Mario Hütte, Susanne Kundmüller-Bianchini, Annette Kustos, Annemarie Nilges, Anja Platz-Schliebs

\title{
Von der Teaching Library zum Lernort Bibliothek
}
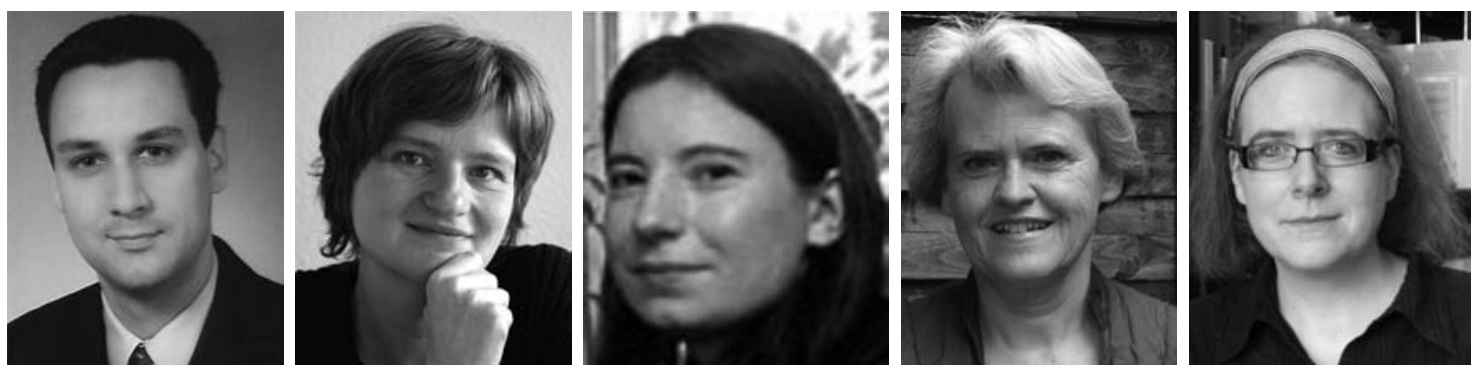

Quereinsteiger und Neulinge der Vermittlung von Informationskompetenz werden grundlegend auf der Basis der vielfältigen praktischen Erfahrungen des Multiplikatorennetzwerks der AG Informationskompetenz NRW über strategische Konzepte, unterschiedliche Schulungsangebote für verschiedene Zielgruppen, Methodik und Didaktik von Schulungsveranstaltungen, Organisation und Infrastruktur sowie Evaluation und Qualitätsmanagement informiert.

Information Literacy: From the Teaching Library to the Learning Centre

Newcomers to the field of teaching information literacy are thoroughly informed about strategic concepts, specific courses for varying target groups, theory and methodology of teaching, organisation and infrastructure as well as evaluation and quality management. The recommendations are based on the wide range of practical experience gathered within the dissemination network of the Project Group Information Literacy NRW.

Tour d'horizon de la maîtrise de l'information

Des débutants en formation à la maîtrise de l'information seront informés sur des conceptions stratégiques, des offres de formation pour des cibles particulières, des modèles méthodologiques et didactiques pour des formations, l'organisation et l'infrastructure ainsi que l'évaluation et le système de management de qualité. Ces recommandations basent sur les expériences pratiques du réseau des multiplicateurs de l'atelier de maîtrise de l'information NRW.

\section{Inhaltsübersicht}

1 Einführung

2 Optimierung der Informationskompetenz als bibliothekspolitisches Ziel: strategisches Konzept und

Schulungsangebot....

2.1 Das strategische Konzept der Bibliothek zur Vermittlung von Informationskompetenz .....

Bologna-Prozess und Schlüsselqualifikationen

2.4 Möglichkeiten der Integration der bibliothekarischen Veranstaltung ins Lehrangebot der Hochschule......

Didaktische Konzepte / Lehr- und Lernformen

3.1 Lehr- und Lernformen im Überblick

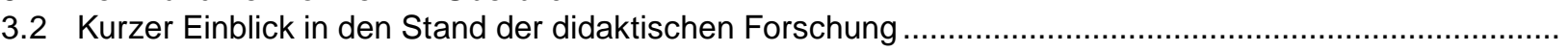

3.3 Erfahrungen aus der Praxis der Vermittlung von Informationskompetenz in nordrhein-westfälischen Hochschulbibliotheken...

3.4 Weitere Tipps für die Praxis

$\begin{array}{ll} & 151\end{array}$

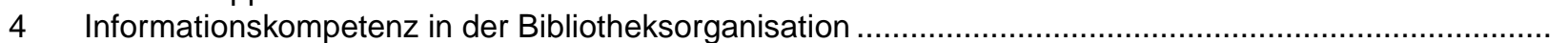

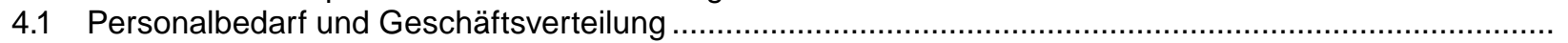

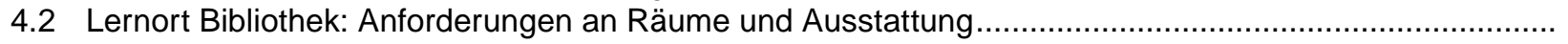

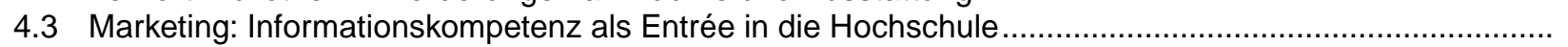

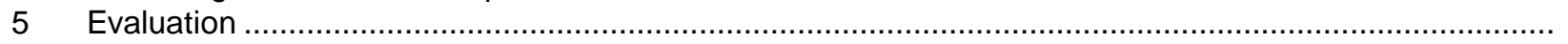

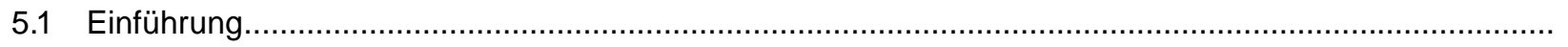

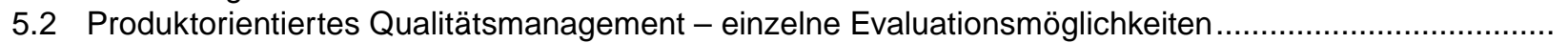

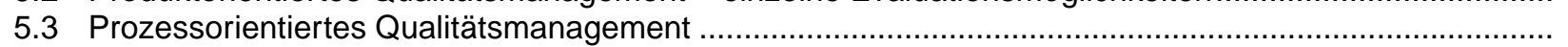
Fazit . 


\section{$1 \quad$ Einführung}

In Deutschland strebte im Zuge von Bologna zunächst jede Bibliothek danach, Veranstaltungen zur Vermittlung von Informationskompetenz anzubieten und möglichst in die Curricula integriert zu werden, um sich in den Kreis der Teaching Libraries einreihen zu können ${ }^{1}$. Dabei wurde die Vermittlung von Informationskompetenz nahezu gleichgesetzt mit der Organisation und Durchführung von Schulungen. Spätestens seit 2008 hat jedoch ein Umdenken eingesetzt ${ }^{2}$. Vermittlung von Informationskompetenz ist viel mehr als Schulung. Es ist eine der Leitideen bibliothekarischen Agierens in den Hochschulen und oberstes Ziel für alle unsere Aktivitäten. Darunter fällt die Auseinandersetzung mit der Optimierung von Nutzeroberflächen in Suchräumen oder im Katalog der Zukunft genauso wie die bibliothekarische Präsenz bei den strukturierten Postgraduierten-Programmen. Der Focus hat sich dabei vom nahezu allwissenden Schulungsbibliothekar als Lehrer des unbedarften Studierenden hin zum Lernenden verschoben. Nicht von oben herunter zu schulen, sondern auf gleicher Augenhöhe dem Lernenden das Lernen zu erleichtern, ist das neue Ziel geworden. Die Teaching Library hat sich zur Learning (Facilitating) Library gewandelt. Das ist auch nicht ohne Auswirkung auf die Organisation der Vermittlung von Informationskompetenz geblieben. Individualisierte und personalisierte Angebote auf Lernplattformen und eigene Zugänge zu Online-Tutorials ermöglichen einen auf die eigenen Bedürfnisse zugeschnittenen Erwerb von Informationskompetenz, und auch die klassischen Schulungsveranstaltungen sollten versuchen, vom Lernstand der einzelnen Teilnehmer auszugehen, statt eine Einheitsveranstaltung für alle anzubieten. Die Bibliothek als Ganzes wird zum Lernort ${ }^{3}$, nicht mehr nur der für die Vermittlung von Informationskompetenz zur Verfügung stehende Schulungsraum. Dies sollte bei der Erarbeitung von Konzepten zur Vermittlung von Informationskompetenz stets mitbedacht werden.

Die hier vorgelegten praktischen Handreichungen zur Vermittlung von Informationskompetenz, die keine Ideallösung vorgeben, sondern ein Bündel an Optionen aufzeigen möchten, richten sich primär an Neulinge in diesem Arbeitsbereich. Sie sollen deren Einarbeitung und der allgemeinen Information über die praktische Ausgestaltung dieser Schlüsselqualifikation im Bibliotheksalltag dienen. Eingeflossen sind die oft jahrelangen Erfahrungen und Erkenntnisse des Multiplikatorennetzwerks der AG Informationskompetenz NRW, deren Arbeit unter dem Motto „Aus der Praxis für die Praxis“ steht. Sie werden je nach Aspekt und Notwendigkeit ergänzt durch den theoretischen Hintergrund und beziehen (inter)nationale Verfahrensweisen und Gepflogenheiten mit ein. Je nachdem, ob die jeweilige Hochschulbibliothek alleine für die Informationsversorgung zuständig ist oder sich diese Aufgabe mit dezentralen Fachbibliotheken teilt, ob es sich um eine große traditionelle Universitätsbibliothek oder eine überschaubare Fachhochschulbibliothek handelt, sind die Lösungsansätze für eine optimale Vermittlung von Informationskompetenz durchaus unterschiedlich. Jede Bibliothek muss für sich ihr strategisches Konzept erarbeiten, daraus ein Schulungskonzept ableiten, sich mit Fragen der Schulungsinhalte, der Didaktik/Methodik und nicht zuletzt mit der Organisation und der Evaluation ihrer Angebote beschäftigen.
2

\section{Optimierung der Informationskompetenz als bibliothekspolitisches Ziel: strategisches Kon- zept und Schulungsangebot}

\subsection{Das strategische Konzept der Bibliothek zur Vermittlung von Informationskompetenz}

Am Beginn aller Überlegungen sollte das strategische Konzept der Bibliothek zur Vermittlung von Informationskompetenz stehen. Die Bibliothek muss für sich klären, welchen Stellenwert sie der Vermittlung von Informationskompetenz strategisch in ihrem Agieren als Dienstleisterin für ihre Hochschule zumisst, und welche Ressourcen personeller und finanzieller Art sowie welche Räumlichkeiten/Ausstattung ihr zur Verfügung stehen. So kann beispielsweise die Optimierung von Informationskompetenz im Rahmen von exzellenter Lehre oberstes Leitziel sein und mit in die meist jährlichen Zielvereinbarungen zwischen Hochschule und Bibliothek aufgenommen werden. Dann sind alle Aktivitäten der Bibliothek darauf abzustimmen und Teile dieses strategischen Konzepts. Dazu gehören die Gestaltung von Suchinstrumentarien (Erarbeiten nutzerfreundlicher Suchoberflächen möglichst als One-stopshop) genauso wie die Ausgestaltung der realen und virtuellen Räumlichkeiten (mehr Bestand im Lesesaal oder mehr Nutzerarbeitsplätze; Präsenz auf der Lernplattform, attraktives Webangebot) und die Organisation von Schulungsveranstaltungen. Für diese Bestandteile innerhalb des Gesamtziels werden dann dem Leitziel untergeordnete Zielvereinbarungen getroffen. Die Arbeitsergebnisse müssen regelmäßig qualitätsüberprüft und sie sollten öffentlichkeitswirksam präsentiert werden.

Viele Bibliotheken beginnen jedoch zunächst in einem ersten Schritt pragmatisch mit der Erarbeitung eines Schulungskonzepts. Hier ist vorab zu klären:

- Welches Personal steht zur Verfügung, und wie kann es synergetisch zusammenarbeiten (Auskunftsbibliothekare, Fachreferenten etc.)

1 Vgl. zur Begriffsdiskussion Nilges, Annemarie und Anneke Thiel: Informationskompetenz im Wandel: Theorie und Praxis. In: Jahrbuch der Heinrich-Heine-Universität 2003. Düsseldorf 2004, S. 513-529.

2 Vgl. z. B. Schoenbeck, Oliver: Leitidee Informationskompetenz. Vorschläge aus der Arbeit der DINI-AG „E-Kompetenzen“. Vortrag, gehalten auf dem 97. Deutschen Bibliothekartag 2008 in Mannheim <http://www.opus-bayern.de/ bib-info/volltexte/2008/470/pdf/DINI_Schoenbeck_InfoKomp. pdf> [Zugriff am 15.4.2009].

3 Zum Konzept der Bibliothek als Lernort vgl. z. B. Gläser, Christine: Die Bibliothek als Lernort - neue Servicekonzepte. In: Bibliothek. Forschung und Praxis 32 (2008) 2, S. 171-182. Weckmann, Hans-Dieter: Macht Lernen in CIP-Pools Spass? Moderne Lernarbeitsplatze an deutschen Hochschulen. In: Bibliothek. Forschung und Praxis 32 (2008) 2, S. 167-170. Schoenbeck, Oliver: Platz schaffen für neue Bedürfnisse - ein neuer Lernort auf alten Flächen. In: Bibliothek. Forschung und Praxis 32 (2008) 2, S. 183-187. Strauch, Thomas: E-Kompetenzentwicklung im öffentlichen Hochschulraum - Herausforderung für zentrale Einrichtungen. In: Bibliothek. Forschung und Praxis 32 (2008) 2, S. $160-167$. 
- Art der Einbringung in die Lehre

- welche Zielgruppen sollen bedient werden (nur Studierende, auch die Forschung, Schüler, Stadtleser etc.)

- fachwissenschaftliche und/oder fachübergreifende Angebote

- Abstimmung auf Auskunftsdienste, Online-Auskunft, E-Learning-Angebote

- Zusammenarbeit mit anderen Institutionen z. B. Rechenzentren, Multimediazentren etc.

- organisatorische Einbindung der Aufgabe in den Geschäftsplan (Stabsstelle, Querschnittsaufgabe etc.).

Vor allen Dingen muss jede Bibliothek für sich klären, ob sie integrativ arbeiten, d. h. Informationskompetenz innerhalb der von den Fächern angebotenen Module vermitteln will, oder additiv, d. h. im Rahmen eines eigenständigen Moduls in eigener Verantwortung. Mit der Hochschule ist zu vereinbaren, ob die Bibliothek bei ihren eigenen Angeboten ECTS-Punkte vergeben darf. Möchte sie das tun, sollte sie sich darüber im Klaren sein, dass sie dann einen hohen personellen Aufwand durch die Korrektur der curricular geforderten Abschlussprüfungen in Form von Klausur oder Hausarbeit auf sich nimmt.

Dies sind nur einige der Aspekte, die vor der Erarbeitung eines Schulungskonzepts als Teil des strategischen Konzepts von der Bibliotheksleitung zu entscheiden sind. Es gibt keinen Königsweg. Infrastruktur, Ansprechpartner in der Hochschule und deren Erwartungen an die Bibliothek sowie deren Selbstverständnis und ihre Ressourcen sind jeweils höchst unterschiedlich, so dass jede Institution in Zusammenarbeit mit ihrer Hochschule ihren eigenen Weg finden muss.

Für das bibliotheksintern entwickelte strategische Konzept ist nun die Akzeptanz in der Hochschule zu suchen. Idealerweise hat man den ganzen Überlegungen eine genaue Bedarfsanalyse der Hochschule vorgeschaltet oder sich mit vertrauten und bewährten Ansprechpartnern innerhalb der Hochschule vorab beraten. Ideal ist es, wenn das Konzept gleich einer breiteren Hochschulöffentlichkeit, z. B. dem um Dekane und Studiendekane erweiterten Rektorat und Studentenvertretern, gemeinsam vorgestellt werden kann. Oft sind jedoch langwierige Vorstellungstermine in den einzelnen Fakultäten und anderen Gremien nötig, um das vermutlich mehrfach zu modifizierende Konzept dann schließlich zu verabschieden $^{4}$. Dieser mühselig erscheinende Prozess hat jedoch auch nützliche Seiten: Die Diskussion um das neue Angebot der Bibliothek bzw. die neue strategische Ausrichtung rücken die Bibliothek als kompetente Partnerin für Lehre und Forschung in den Mittelpunkt und zwingen die Hochschule zur Auseinandersetzung mit dieser für sie so wichtigen Einrichtung.

\subsection{Bologna-Prozess und Schlüsselqualifikatio- nen}

Die so genannten konsekutiven Studiengänge (B.A., M.A.), deren Konzeption, Akkreditierung und Reakkreditierung bieten den Bibliotheken konkrete Ansätze der Positionierung im Bereich Vermittlung von Informationskompetenz. Um sich besser vergegenwärtigen zu können, vor welche Konsequenzen und Herausforderungen der Bologna-Prozess nicht nur die Hochschulen, sondern auch deren Bibliotheken stellte und immer noch stellt, aber auch welche
Chancen sich daraus für sie bieten, sollen im Folgenden zunächst die Hintergründe der Strukturveränderungen kurz beleuchtet werden.

Die Hauptziele des Bologna-Prozesses von 1999 umfassen die Förderung von internationaler Wettbewerbsfähigkeit, von Beschäftigungsfähigkeit sowie räumlicher und kultureller Mobilität. Um diese zu realisieren, werden seither an den europäischen Hochschulen mit einer Umsetzungsfrist bis 2010 vergleichbare Bachelor- und Master-Studiengänge geschaffen und ein Leistungspunktesystem, nämlich das European Credit Transfer System (ECTS), eingeführt ${ }^{5}$. Die Leistungspunkte sind ein Maß für den studentischen Arbeitsaufwand, den so genannten Workload. Dieser umfasst neben dem Besuch der Lehrveranstaltungen auch die Zeit für deren Vor- und Nachbereitung, für Prüfungen und das Selbststudium. Dabei entspricht ein ECTS-Punkt einem Workload von 30 Stunden. Die Regelstudienzeit im Bachelor-Studiengang beträgt drei bis vier Jahre, wobei - je nach Studienfach mindestens 180 bzw. 240 ECTS-Punkte nachzuweisen sind. Der daran anschließende Master-Studiengang ist angelegt auf ein bis maximal zwei Jahre, in denen weitere 90 bis 120 ECTS-Punkte zu erlangen sind. Nach Erhebungen des Bundesministeriums für Bildung und Forschung (BMBF) wurden in Deutschland über die Hälfte der Bachelor- und Master-Studiengänge neu entwickelt, die anderen entstanden bei der inhaltlichen und strukturellen Reform des bestehenden Studienangebots. Ein Studium besteht aus mehreren Modulen, wobei unter einem Modul eine inhaltlich und zeitlich abgeschlossene Lehr- und Lerneinheit zu verstehen ist, die auch mehrere Semester und unterschiedliche Lehrveranstaltungen umfassen kann. Die Module können verpflichtend oder Teil des Wahlbereichs sein. Jedes von ihnen wird mit einer Modulabschlussprüfung abgeschlossen und mit einer bestimmten Anzahl von ECTS-Punkten und gegebenenfalls Noten versehen.

4 So war beispielsweise ein entsprechendes Projekt für die Anglistik, Germanistik und Romanistik an der Bergischen Universität Wuppertal mit einer Vielzahl von Fachvertretern abzustimmen. Vgl. Blume, Peter und Anja Platz-Schliebs: Grundlagen der Literaturrecherche und -beschaffung für Studienanfänger der Sprach- und Literaturwissenschaften. Ein neues Schulungsangebot der Universitätsbibliothek Wuppertal. In: Bibliotheksdienst 42 (2008) 11, S. 1 222-1 237.

5 Zu Hintergrund, Inhalt sowie Stand der Umsetzung des Bologna-Prozesses in Deutschland vgl. Bundesministerium für Bildung und Forschung: Der Bologna-Prozess $<$ http://www.bmbf.de/de/3336.php> [Zugriff am 15.4.2009]. Für eine Übersicht der neuen Studiengänge und Abschlüsse s. Hessisches Ministerium für Wissenschaft und Kunst: Studien- \& Berufswahl <http://www.studienwahl.de> [Zugriff am 15.4.2009]. Zur Implementierung von Informationskompetenz-Veranstaltungen in Bachelor-Studiengängen vgl. die Ergebnisse des Workshops „Informationskompetenz als Schlüsselqualifikation in den Bachelor-Studiengängen" der AG Informationskompetenz am 11. März 2004 in Bonn <http://www.ulb.uni-bonn.de/informationskompetenz/ protokolle/ws-ba_040311/ws-ba_040311_protokoll.pdf> [Zugriff am 15.4.2009]. 
Zur Erhöhung der beruflichen Relevanz der neuen Bachelor- und häufig auch der Master-Studiengänge liegt ein besonderer Schwerpunkt des Studiums auf dem Erwerb von Schlüsselqualifikationen wie der Sozial-, Methoden-, Individual-, Handlungs- und Medienkompetenz. Schlüsselqualifikationen sind folglich kein spezifisches Fachwissen, sondern es handelt sich dabei vielmehr um Fähigkeiten, die den kompetenten Umgang mit fachlichem Wissen ermöglichen $^{6}$. In den Bereich der Schlüsselqualifikationen fällt auch die Bibliotheks- und Informationskompetenz, die den Bachelor- und Master-Studierenden systematisch vermittelt werden sollte, um ihnen die zielgerichtete Recherche nach wissenschaftlichen Informationen und deren effiziente Verwendung zu ermöglichen ${ }^{7}$. Insbesondere in diesem Kontext können sich die Hochschulbibliotheken mit ihren Lehrveranstaltungen aktiv beteiligen. Dies wird sogar ausdrücklich vom Wissenschaftsrat gefordert: „Die Vermittlung von Medien- und Informationskompetenz als Schlüsselqualifikation kann (...) nicht allein von den Fachwissenschaftlern geleistet werden. Der Verbesserung der Nutzerkompetenz (information literacy) muss die Bibliothek der Hochschule durch das aktive Angebot geeigneter Benutzerschulungen verstärkt Rechnung tragen."8

Die Entscheidung für oder gegen die Vermittlung von Informationskompetenz in einem eigenständigen Modul oder innerhalb der von den Fächern angebotenen Module ist, wie oben ausgeführt, Teil der strategisch-bibliothekspolitischen Grundentscheidungen.

\subsection{Arten von Schulungsveranstaltungen}

Die Konzeption einer auf den Bachelor- oder Master-Studiengang abgestimmten bibliothekarischen Schulungsveranstaltung ist in erster Linie abhängig von der Zielgruppe, die mit dem Schulungsprogramm erreicht werden soll, und zwar von

- der fachlichen Ausrichtung (Geistes-, Gesellschafts-, Naturwissenschaften, Life Sciences oder Ingenieurwissenschaften)

- dem Niveau (Grundlagen, Aufbau oder Vertiefung)

- dem inhaltlichen Schwerpunkt (Bibliotheksbenutzung, Suchstrategien, einzelne Kataloge und Fachdatenbanken etc.).

In der Regel müssen sich Studierende der Geistes- und Gesellschaftswissenschaften in einem wesentlich früheren Abschnitt ihres Studiums mit fachlichen Datenbanken auseinandersetzen als ihre Kommilitoninnen und Kommilitonen aus den natur- und ingenieurwissenschaftlichen Fächern. Denn bereits zu Beginn des Grundstudiums sind sie dazu aufgefordert, Referate zu halten, Protokolle und nicht zuletzt - Hausarbeiten zu schreiben. Nicht selten verzichten die Dozentinnen und Dozenten in ihren Seminaren inzwischen darauf, ihren Studierenden vorbereitete Literaturlisten auszuhändigen, da auch sie der Kompetenz zur eigenständigen Informations- und Literaturrecherche sowie deren Auswahl einen hohen Stellenwert beimessen. Ein zielgruppengerechtes Schulungsangebot muss folglich in den Geistes- und Gesellschaftswissenschaften idealerweise bereits im frühen Grundstudium angeboten werden, während der fachbezogene Bedarf für Studierende der natur- und ingenieurwissenschaftlichen Fächer in der Regel erst in einem späteren Studienabschnitt, spätestens zu ihrer Studien- und Abschlussarbeit, gegeben ist.
Neben der fachlichen Ausrichtung ist die Schulung auf das Niveau der jeweiligen Benutzergruppe anzupassen. So unterscheidet sich der Informationsbedarf von Studienanfängern erheblich von dem fortgeschrittener Studierender, die sich auf die Bachelor- bzw. Master-Arbeit vorbereiten, oder gar dem hochwissenschaftlichen von Doktoranden.

Eng verbunden mit der fachlichen Ausrichtung und dem Niveau sind die inhaltlichen Schwerpunkte der bibliothekarischen Schulungen. Tab. 1 stellt eine Matrix an Inhalten bibliothekarischer Schulungen dar, die nach Niveau (Grundlagen, Aufbau vs. Vertiefung) und fachlicher Relevanz (fachübergreifend versus fachbezogen) unterschieden werden.

Grundlagen-, Aufbau- und Vertiefungskurse bauen inhaltlich aufeinander auf. Idealerweise werden sie von den Studierenden in dieser Abfolge besucht. So können in den Aufbaukursen die grundlegenden Inhalte bereits vorausgesetzt oder aber kurz wiederholt werden. Für die erfolgreiche Gestaltung der Vertiefungskurse schließlich sollten spezifische Vorkenntnisse unerlässlich sein.

Bei den angeführten Inhalten handelt es sich um eine offene Zusammenstellung, die den gängigen und heutzutage aktuellen fachübergreifenden und fachbezogenen Informationsbedarf der Studierenden widerspiegelt. Selbstverständlich sind weitere bibliothekarische Schulungsinhalte als die genannten denkbar, die sich in das Lehrangebot der Hochschule einbringen ließen. Das Niveau der Inhalte kann im Hinblick auf die zu schulende Gruppe variiert werden, um aus Grundkursen Aufbaukurse zu gestalten und umgekehrt. So würden in einem Aufbaukurs den formalen Suchtechniken neben den grundlegenden Boole'schen Operatoren auch datenbankspezifische Kontextoperatoren und gegebenenfalls die jeweilige Retrievalsprache behandelt. Ebenso können die meisten Inhalte - je nach Bedarf - fachübergreifend oder fachbezogen angeboten werden. Ein Beispiel dafür ist eine Schulung zum Aufbau und Umgang mit Datenbanken, die sich fachübergreifend auf das Phänomen Datenbank allgemein und fachbezogen z. B. auf die Benutzung der MLA International Bibliography beziehen kann. Die in den Tabellenfeldern aufgelisteten Inhalte können jeweils zum Gegenstand eigener Schulungsveranstaltungen oder aber auch zielgruppenspezifisch miteinander kombiniert werden.

6 Für eine detaillierte Beschreibung der Schlüsselqualifikationen vgl. z. B. Sidler, Fredy: Studiengangsprofile. Die Konzeption outcome-orientierter Studiengänge. In: Hanft, Anke und Isabel Müskens ( $\mathrm{Hg}$.): Bologna und die Folgen für die Hochschulen. Bielefeld 2005, S. 28-51.

7 Für die an Vorarbeiten anglo-amerikanischer Bibliotheken und Hochschulen anknüpfenden Standards der Informationskompetenz für Studierende s. Netzwerk Informationskompetenz Baden-Württemberg (NIK-BW) (Hg.): Standards der Informationskompetenz für Studierende <http:// www.informationskompetenz.de/fileadmin/DAM/documents/ Standards\%20der\%20Inform_88.pdf> [Zugriff am 15. 4. 2009]. Verantwortlich für die Weiterentwicklung nationaler Standards ist die DBV-Dienstleitungskommission.

8 Wissenschaftsrat ( $\mathrm{Hg}$.): Empfehlungen zur digitalen Informationsversorgung durch Hochschulbibliotheken. Greifswald 2001 <http://www.wissenschaftsrat.de/texte/4935-01. pdf> [Zugriff am 15.4.2009], S. 36. 
Tabelle 1: Mögliche Inhalte bibliothekarischer Schulungsveranstaltungen

\begin{tabular}{|c|c|c|c|}
\hline & \multicolumn{2}{|l|}{ Fachliche Relevanz } \\
\hline & & fachübergreifend & fachbezogen \\
\hline \multirow[b]{3}{*}{ 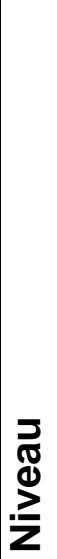 } & $\begin{array}{l}\text { Grund- } \\
\text { lagen }\end{array}$ & $\begin{array}{l}\text { - Bibliotheksbenutzung (Aufstellungssystematik, Be- } \\
\text { nutzerkonto etc.) } \\
\text { - Literatursuche im Bibliothekskatalog } \\
\text { - Formale Suchtechniken (Suchmodi, Operatoren, } \\
\text { Trunkierung etc.) } \\
\text { - Zielgerichtete Internetrecherche, Suchmaschinen }\end{array}$ & $\begin{array}{l}\text { - Bearbeitung von Literaturlisten (Primär- und Se- } \\
\text { kundärliteratur, Publikationsformen etc.) } \\
\text { - Umgang mit den einschlägigen bibliographischen } \\
\text { Fachdatenbanken } \\
\text { - Kenntnis fachlich relevanter Volltextdatenbanken }\end{array}$ \\
\hline & Aufbau & $\begin{array}{l}\text { - Inhaltliche Suchstrategien (Thesauri, thematische } \\
\text { Felder, Schlagwörter etc.) } \\
\text { - Literaturbeschaffung (Fernleihe, DigiBib etc.) } \\
\text { - Benutzung eines Literaturverwaltungsprogramms }\end{array}$ & $\begin{array}{l}\text { - E-Journals in der Elektronischen Zeitschriftenbibli- } \\
\text { othek } \\
\text { - Umgang mit E-Books } \\
\text { - Fachinformationen im Internet (Wörterbücher, Por- } \\
\text { tale, Virtuelle Fachbibliotheken etc.) }\end{array}$ \\
\hline & $\begin{array}{l}\text { Vertie- } \\
\text { fung }\end{array}$ & $\begin{array}{l}\text { - Social Software (Weblogs, Wikis, Collaborative } \\
\text { Tagging) } \\
\text { - Elektronisches Publizieren } \\
\text { - Plagiarismus }\end{array}$ & $\begin{array}{l}\text { - Differenzierung nach individuellem Informations- } \\
\text { bedarf (Examensarbeit, Recherche für eine Dok- } \\
\text { torarbeit etc.) }\end{array}$ \\
\hline
\end{tabular}

Ist die Zielgruppe nach fachlicher Ausrichtung, Niveau und inhaltlichem Schwerpunkt definiert, muss die organisatorische Form der bibliothekarischen Schulungsveranstaltung gewählt werden.

\subsection{Möglichkeiten der Integration der bibliothekari- schen Veranstaltung ins Lehrangebot der Hoch- schule}

Bei den bibliothekarischen Schulungsveranstaltungen sind zunächst die Angebote, die speziell auf die verschiedenen Studiengänge des Hochschulstudiums ausgerichtet sind, von den weiteren Angeboten zu unterscheiden. Dabei handelt es sich um allgemeine Einführungen in die Bibliotheksbenutzung (Katalog, E-Books etc.), auch für Zielgruppen wie Schüler und Senioren, sowie um sonstige Schulungen zur Vermittlung von Informationskompetenz, die jeweils fachübergreifend oder fachbezogen sein können. Einerseits werden allgemeine Schlüsselkompetenzen wie der richtige Umgang mit dem Internet oder den Strategien zur Recherche vermittelt, andererseits auch fachspezifische Beratungen für fortgeschrittene Studierende und Examenskandidaten und spezielle Schulungen für Tutoren angeboten. Zwar sind diese Bibliotheksveranstaltungen inhaltlich auf das Studium ausgerichtet, jedoch ohne Verankerung in einem eigenen oder fachlichen Curriculum. Sie richten sich an alle Studiengänge, sind in der Regel ein- oder mehrstündig und schließen gegebenenfalls mit einem Teilnahmenachweis ab. Abb. 1 stellt eine Typologie bibliothekarischer Schulungsveranstaltungen dar ${ }^{9}$.

Die bibliothekarischen Schulungsveranstaltungen können auf zwei Wegen in die Wahl- oder (Wahl)Pflichtangebote der Studiengänge eingebunden werden, nämlich als eigenständiges Angebot der Bibliothek (= additiv) oder in Form eines obligatorischen Teils innerhalb eines Angebots des Fachs (= integrativ). Da in den Pflichtveranstaltungen die grundlegenden Inhalte des jeweiligen Studiums vermittelt werden, sind sie von den Studierenden obligatorisch zu besuchen. Zu unterscheiden sind die „reinen“ Pflichtveranstaltungen von so genannten Wahlpflichtveranstaltungen, bei denen die Studierenden aus einem Kontingent von Pflichtveranstaltungen auswählen können. Die obligatorischen Veranstaltungen müssen gemäß der Studienordnung im Grund- bzw. Hauptstudium durch eine Prüfungsleistung (Klausur, Hausarbeit o. ä.) erfolgreich abgeschlossen werden. Wahlveranstaltungen sind von den Studierenden frei wählbar. Sie dienen dazu, das Wissen zu vertiefen und ermöglichen es, Schwerpunkte nach Interesse zu legen. Um kalkulierbare Teilnehmerzahlen zu erreichen, ist folglich die Einbindung eines additiven oder integrativen Angebots in ein Pflicht- oder auch Wahlpflichtmodul optimal.

Additive Veranstaltungen können fachübergreifend oder fachbezogen sein:

- Fachübergreifende Veranstaltungen sind ohne konkrete fachbezogene Einbindung in den Studiengang. Sie befinden sich zumeist im so genannten Optionalbereich ${ }^{10}$.

- Fachbezogene Veranstaltungen sind mit eigenem Curriculum in den Bachelor- oder Master-Studiengang eingebunden und inhaltlich speziell für das jeweilige Fachgebiet konzipiert.

Beide Veranstaltungsarten, bei denen gegebenenfalls ECTS-Punkte gemäß der Studienordnung vergeben werden, können entweder semesterlang oder als Blockveranstaltung angeboten werden.

9 Abb. 1 ist ein Modell der möglichen bibliothekarischen Schulungsveranstaltungen und erhebt keinen Anspruch auf Vollständigkeit. Bei der Vielfalt an Schulungs- und Einbindungsformen ins Lehrangebot der Hochschule wurden hier nur die typischen berücksichtigt. Ausführliche Fallbeispiele von Bibliotheken aus unterschiedlichen Bundesländern sind auf dem zentralen Portal <www.informationskompetenz.de $>$ unter dem Menüpunkt, Regionen` gegeben. Siehe hierzu auch Sühl-Strohmenger, Wilfried: Vermittlung der Schlüsselqualifikationen Informations- und Medienkompetenz in den neuen Studiengängen. Ziele, Anforderungen, Konzepte, Strategien - am Beispiel ausgewählter Hochschulbibliotheken (UB Freiburg u.a.). HIS-Workshop Der Beitrag der Bibliotheken zum Bologna-Prozess. Hannover, 22. Februar 2007 $<$ http://www.his.de/publikation/seminar/bibliotheken/Vermittlung.pdf $>$ [Zugriff am 09.12.2008].

${ }^{10}$ Statt Optionalbereich findet sich auch die Bezeichnung „BOK-Bereich“, wobei das Akronym BOK für „BerufsfeldOrientierte-Kompetenzen" steht. 


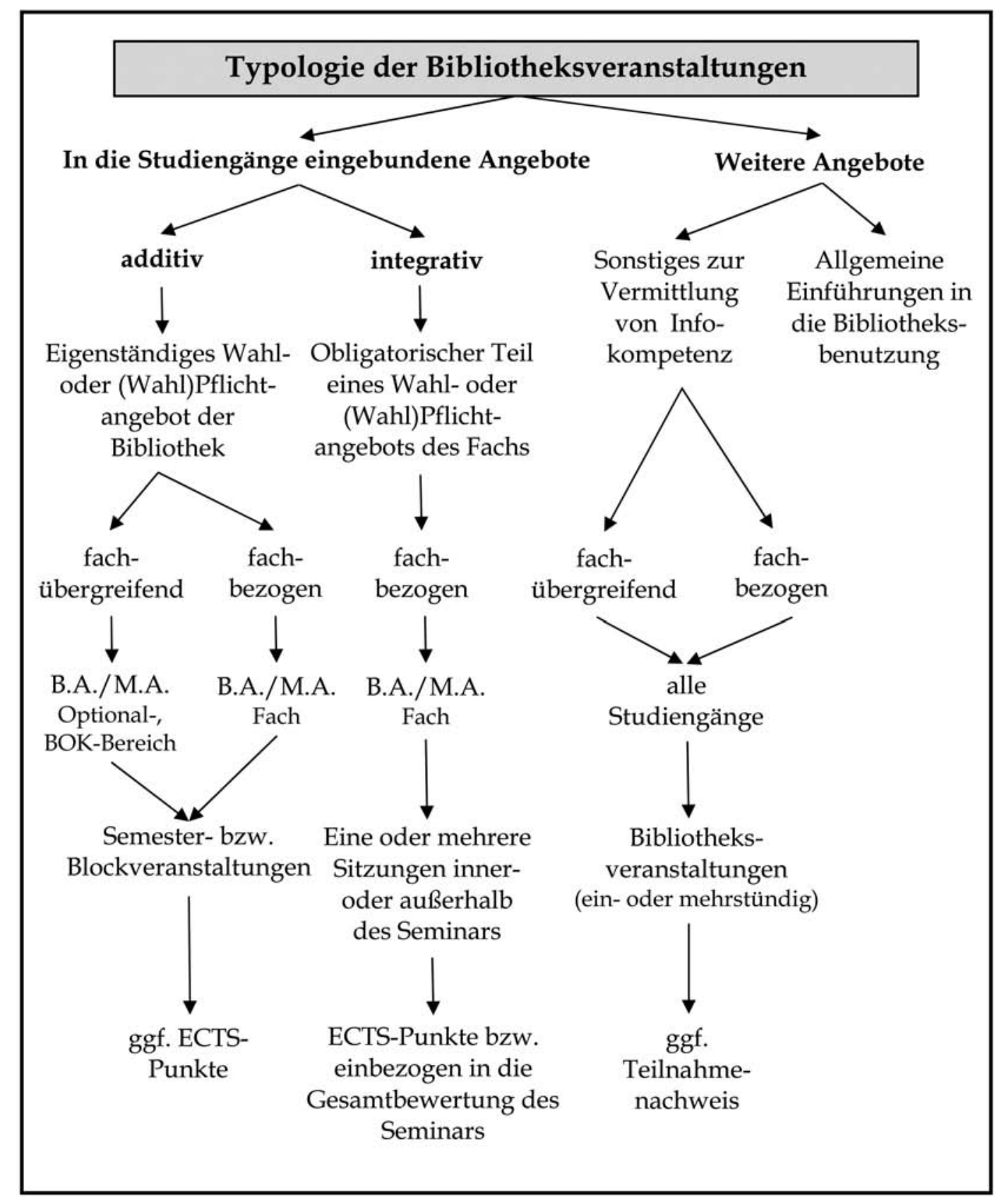

Abb. 1: Typologie bibliothekarischer Schulungsveranstaltungen

Integrative Veranstaltungen sind in einzelne Lehrveranstaltungen eines Studiengangs obligatorisch eingebunden. In diesem Kontext sind sie in der Regel fachbezogen. Die Anzahl der Sitzungen im bzw. außerhalb des Seminars erfolgt nach Absprache mit dem jeweiligen Dozenten. Ebenso können die ECTS-Punkte anteilmäßig auf die bibliothekarische Veranstaltung umgerechnet bzw. in die Gesamtbewertung des Seminars einbezogen werden. In welchem Rahmen eine bibliothekarische Schulungsveranstaltung in das Lehrangebot integriert wird - als Wahl- oder (Wahl)Pflichtangebot - und in welchem zeitlichen Umfang, ist von verschiedenen Faktoren abhängig. Der personelle und organisatorische Rahmen der Durchführbarkeit einer Veranstaltung seitens der Bibliothek, der ebenfalls im übergeordneten strategischen Konzept berücksichtigt sein muss, wird im vierten Teil dieses Aufsatzes diskutiert.
Nachdem Rahmen und Inhalt der geplanten Schulungsangebote abgesteckt sind, muss als nächstes die Entscheidung getroffen werden, wie vermittelt werden soll.

\section{3}

\section{Didaktische Konzepte / Lehr- und Lernformen}

\subsection{Lehr- und Lernformen im Überblick}

Schulungsprogramme variieren in ihren Inhalten und Lernzielen, in ihren Adressatenkreisen und in der Art ihrer Durchführung. Je nachdem, ob es sich um einführende oder vertiefende Veranstaltungen handelt, ob sie einmal oder mehrfach stattfinden, ob sie sich an viele oder wenige Teilnehmer richten, ob die Zielgruppe homogen oder heterogen ist, kommen unterschiedliche Methoden zum Einsatz. Häufig eingesetzt werden dabei: 


\section{Führungen}

Rundgänge durchs Haus werden meist für Studienanfänger und Schüler angeboten und dienen einer ersten Orientierung innerhalb der Bibliothek. Die Teilnehmer erhalten einen unmittelbaren Eindruck von den Räumlichkeiten und Dienstleistungen der Einrichtung und können Fragen im direkten Gespräch mit dem Führenden klären.

\section{Vorträge und Demonstrationen}

Die Teilnehmer erhalten schnelle, strukturierte Informationen zur Bibliothek und ihrer Benutzung, entweder in seminarähnlicher Form oder im Rahmen einer Vorlesung. Mit dieser Form können auch größere Zielgruppen erreicht und auf einen einheitlichen Informationsstand gebracht werden.

\section{Präsentationen vor Ort (Roadshows)}

Die Bibliothekare stellen den Adressaten vor Ort (z. B. in der Mensa, vor den Hörsälen, im Rahmen von Erstsemestertagen etc.) ihr Angebot oder ihre Dienstleistung vor, in der Regel ist dies auch als eine Art Werbemaßnahme zu verstehen. Das Hochschulbibliothekszentrum in Köln beispielsweise bietet vielfältige Unterstützung bei der Organisation und Durchführung von Roadshows zur Digitalen Bibliothek.

\section{Übungen}

Die eher theoretischen Vorträge oder Präsentationen werden oft durch praktische Übungen ergänzt, die entweder in Einzel- oder in Gruppenarbeit zu bewerkstelligen sind. Lernende erhalten so die Möglichkeit, selbst aktiv zu werden und den Lernerfolg zu überprüfen. Besondere Formen von Übungen sind spielerische Formen wie z. B. die Bibliotheksrallye oder das von DANNENBERG entwickelte Karussel|11.

\section{Schriftliche Materialien}

Merkblätter und Broschüren geben in knapper Form Anleitung zur Benutzung verschiedener Bereiche der Bibliothek. Für bestimmte Gebiete stehen auch umfangreichere Lehr- und Handbücher zur Verfügung (z. B. bei Datenbanken). Insbesondere die kürzer gefassten Materialien haben den Vorteil, dass sie ohne großen Aufwand zu erstellen sind und in großen Stückzahlen verbreitet werden können. Für den Lernenden bieten sie die Möglichkeit, sich selbstbestimmt sowie zeit- und ortsunabhängig mit ihrem Inhalt zu beschäftigen. Als Begleitmaterial für Schulungsprogramme und zur Information am Benutzungsort spielen diese eher konventionellen Materialien daher nach wie vor eine große Rolle.

\section{Computergestützte Methoden}

Seit den 80er Jahren wird als weitere Form der Selbstunterweisung auch der computer-gestützte Unterricht eingesetzt (Computer Based Training oder Computer Aided Instruction). Hinter dieser Schulungsform verbirgt sich die Idee, durch die Kombination verschiedener Elemente (Text, Ton, Graphiken, Interaktionen, unterschiedliche Lernlevels etc.) Lernvorgänge computertechnisch zu unterstützen und attraktiver zu gestalten ${ }^{12}$. Für Schulungsbibliothekare bedeutet die elektronische Weiterentwicklung und Verbesserung von Selbstlernmaterialien einerseits eine Entlastung, andererseits ist die Erstellung und Pflege solcher Materialien für die Bibliothek mit Aufwand und Kosten verbunden.

\section{Online-Tutorien}

Gleiches gilt auch für die modernste Variante von Selbstlernmaterialien, die internetbasierten Tutorien. Ebenso wie ihre konventionelleren Vorgänger bieten sie Vorzüge wie zeit- und ortsunabhängige Bedienbarkeit, individuelle Lerngestaltung (z. B. was die Lerngeschwindigkeit oder die Wahl des Schwierigkeitsgrads angeht), Erfolgskontrolle durch Tests und Ähnliches, gleichzeitig ergeben sich durch die verbesserte Technologie Möglichkeiten, das aktive Lernen durch Erkunden und Entdecken, durch praktische Anwendungen und bedarfsbezogene Aufgabenstellungen noch intensiver zu fördern ${ }^{13}$. Auch Nachteile wie die relativ schematische Vermittlung und die geringe Interaktivität bei herkömmlichen computergestützten Programmen können in Online-Tutorien durch Komponenten wie Chat, E-Mailing, Foren und neuerdings auch durch so genannte „Web 2.0“-Elemente ausgeglichen werden ${ }^{14}$. Die Technik allein macht allerdings noch kein gutes Tutorium aus, viel hängt letztlich von der didaktischen Konzeption und Ausgestaltung $\mathrm{ab}^{15}$. All dies stellt hohe Ansprüche an die Entwickler und Betreuer solcher Programme und bindet Personalkapazitäten, so dass eine wirkliche Entlastung der Schulungsbibliothekare nur mit Einschränkung gegeben ist. Dennoch wird die Entwicklung geeigneter Online-Programme als wichtige Aufgabe für die Zukunft gesehen, vor allem im Zusammenhang mit der Einführung der neuen Bachelor-Studiengänge an deutschen Universitäten, die eine erhebliche Ausweitung bibliothekarischer Schulungsaktivitäten mit sich bringen können. Da es vor allem kleineren Bibliotheken kaum möglich sein wird, jeweils ein eigenes Produkt zu entwickeln und zu betreuen, sind auch hier Kooperationen sinnvoll und wichtig (siehe z. B. das Online-Tutorial Informationskompetenz, das von

11 Vgl. das „Karussell“ innerhalb des von Detlev Danneberg konzipierten „Lernsystems Informationskompetenz" < http:// www.lik-online.de/ppt/karussell/frame.html\#slide0027.html> [Zugriff am 09.12.2008].

12 Vgl. Feierabend, Jörg und Winfried Gödert: Computer Based Training zur Einführung in die Benutzung elektronischer Informationsmittel. In: Wefers, Sabine (Hrsg.): Die Herausforderung der Bibliotheken durch elektronische Medien und neue Organisationsformen / 85. Deutscher Bibliothekartag in Göttingen (Zeitschrift für Bibliothekswesen und Bibliographie: Sonderheft; 63). Frankfurt a. M. 1996, S. 39-50, hier S. 41.

${ }^{13} \mathrm{Vgl}$. Rauchmann, Sabine: Die Vermittlung von Informationskompetenz in Online-Tutorials: eine vergleichende Bewertung der US-amerikanischen und deutschen Konzepte: Diplomarbeit. In: Fuhlrott, Rolf (Hrsg.): Innovationsforum 2003. Informationskompetenz (B.I.T online: Innovativ; 5). Wiesbaden 2003, S. 189-285, hier S. 221.

${ }^{14} \mathrm{Vgl}$. Reynolds, Leslie J.: Model for a Web-Based Information Literacy: Course Design, Conversion and Experiences. In: Science and Technology Libraries 19 (2001) 3/4, S. 165-178.

${ }^{15}$ Sabine Rauchmann hat in ihrer Diplomarbeit zahlreiche amerikanische und deutsche Tutorials miteinander verglichen und sie einer Bewertung unterzogen, so z. B. das „Texas Information Literacy Tutorial2" (TILT) der Universität Texas oder den „Schlauen Det“ der Fachhochschule Hamburg. Vgl. Rauchmann 2003 (Anm. 13), hier S. 223 ff. 
Hochschulbibliotheken in Nordrhein Westfalen gemeinsam entwickelt wurde) ${ }^{16}$.

\subsection{Kurzer Einblick in den Stand der didaktischen Forschung}

Die Didaktik, eine Unterdisziplin der Pädagogik, umfasst Theorie und Praxis des Lehrens und Lernens. Im Gegensatz zu den Fachdidaktiken der einzelnen Wissenschaftsfächer beschäftigt sich die Allgemeine Didaktik dabei fachübergreifend mit der Gestaltung von Lernangeboten sowie der Lerntechnik ${ }^{17}$.

Innerhalb der Didaktik waren in den letzten Jahren und Jahrzehnten behaviouristische Lerntheorien heftiger Kritik ausgesetzt und wurden weitgehend durch konstruktivistische Lernideen abgelöst.

Der Behaviourismus stellt die Lehrkraft als Autorität und die Wiedergabe von Informationen sowie das Memorieren von Fakten in den Vordergrund. Individuelle Faktoren werden völlig vernachlässigt, vielmehr wird verhaltenspsychologisch einem „Lehrinput“ generell ein „Lernoutput" als Reaktion unterstellt ${ }^{18}$.

Demgegenüber stellen konstruktivistische Lernideen den Lernenden in den Vordergrund. Lernen wird als ein individueller, selbstgesteuerter Prozess betrachtet, der je nach Vorkenntnissen und Vorerfahrungen sehr unterschiedlich verlaufen kann. Der Lernende soll weitgehend eigenständig Lösungen entwickeln anstatt Fakten auswendig zu lernen. Aus konstruktivistischer Sicht wird die Aufgabe einer Lehrkraft in erster Linie als die eines Coach gesehen, der den individuellen Lernprozess anregen und unterstützen sowie für die richtige Lernumgebung sorgen soll.

Als wesentlich für den Wissenserwerb und damit für den Lernerfolg wird die Lernmotivation, insbesondere die intrinsische Lernmotivation, betrachtet. Gefördert werden kann die Eigenmotivation des Lernenden z. B. durch Realitätsnähe und Anwendungsbezug des Lerngegenstandes, Methodenvielfalt, Formulierung von Lernzielen, Eigenaktivität in Form von Übungen oder Gruppenarbeit $^{19}$. Insbesondere das handlungsorientierte Lernen, das "Learning by doing" trägt in hohem Maße dazu bei, dass sich der Lernende die Lerninhalte langfristig einprägt und ist somit ein wichtiger Faktor für den Lernerfolg.

\subsection{Erfahrungen aus der Praxis der Vermittlung von Informationskompetenz in nordrhein-westfäli- schen Hochschulbibliotheken}

Gemäß der Lernideen konstruktivistischer Didaktik bestand im Kreis der Bibliotheken, die im Rahmen der AG Informationskompetenz zusammenarbeiten, schon früh Einigkeit darüber, nach Möglichkeit Veranstaltungen anzubieten, die sich durch aktivierende Unterrichtsformen und einen hohen Anteil praktischer Übungen auszeichnen ${ }^{20}$.

Nahezu alle Konzepte der nordrhein-westfälischen Hochschulbibliotheken sind mittlerweile modularisiert und auf einzelne Zielgruppen ausgerichtet. Modularisierung, d. h. Veranstaltungen, die hinsichtlich des inhaltlichen Niveaus aufeinander aufbauen, und Zielgruppenorientierung sind dabei Eckpunkte eines individuellen Informationskompetenz-Konzeptes, die sich auch in der Didaktik niederschlagen. Eine Aufteilung des Gesamtangebots bewirkt eine Reduzierung des Lernstoffs in den Einzelmodulen. Da- mit bleibt mehr Zeit für didaktisch wertvolle Übungsaufgaben. Die Zielgruppenorientierung führt zu einer stärkeren (in der Regel fachlichen) Fokussierung des Stoffes, bietet Platz für Übungsbeispiele mit mehr Anwendungsbezug und führt damit tendenziell zu einer höheren Motivation bei den Teilnehmern in Form von Interesse und Aufmerksamkeit.

Die Übernahme ein bis mehrerer Sitzungen in fachwissenschaftlichen Veranstaltungen der von den Fächern angebotenen Module wird gegenüber eigenständigen Veranstaltungen oder Modulen in vielen Fällen bevorzugt. Die integrative Vermittlung von Informationskompetenz hat den Vorteil, dass entsprechende Veranstaltungen hinsichtlich Anzahl, Lernstand und Motivation besser kalkulierbar sind, was diesen Typus ressourceneffizienter für die Bibliothek macht. Didaktisch vorteilhaft an dieser Form der Veranstaltungsorganisation ist der homogene Teilnehmerkreis aus meist einem Semester eines Studiengangs, was wiederum eine gezielte Behandlung der Informationsressourcen eines Faches und Anforderungsniveaus in Verbindung mit passenden Übungen ermöglicht.

Alle oben genannten Unterrichtsformen werden, an den meisten Bibliotheken jedoch nur in Auswahl, erfolgreich in Nordrhein-Westfalen durchgeführt. Ergänzt und flankiert werden diese Veranstaltungstypen mancherorts auch durch Eingangsdiagnosen zum Lernniveau der Teilnehmer, Lernstandskontrollen zur Erfolgsermittlung und durch E-Learning-Elemente. Dabei kommt auch innerhalb des Gesamtlernangebots in vielen Bibliotheken nicht nur eine Unterrichtsform zum Einsatz.

Welches methodische Konzept kommt nun für die jeweilige Bibliothek in Frage? Dies hängt stark von den örtlichen Gegebenheiten ab: Die wichtigsten Faktoren hierbei sind das Zeitdeputat der Teilnehmer, die für Schulungsaktivitäten vorhandenen Personalressourcen und die räumlichtechnische Infrastruktur.

So führt der meist enge Unterrichtsplan der konsekutiven Studiengänge vielfach zur Beschränkung von Informationskompetenz-Veranstaltungen auf eine Doppelstunde (90 Minuten). Stehen dem Schulungsteam selbst dagegen nicht ausreichend Zeitressourcen zur Verfügung, kann das zu Einschränkungen bei Breite und Tiefe - und damit auch bei der methodischen Form - des Veranstaltungsangebots der Bibliothek führen.

Schließlich ist es inzwischen, da fast alle Recherchesysteme und viele Informationsressourcen digital vorliegen, kaum mehr möglich, Veranstaltungen mit praktischen Übungen zu konzipieren, ohne eine ausreichende Anzahl

16 S. Online-Tutorial Informationskompetenz <http://www. informationskompetenz.de/regionen/nordrhein-westfalen/ online-tutorial-informationskompetenz/> [Abruf am 3.11.2008].

17 Vgl. Riedl, Alfred: Grundlagen der Didaktik. Wiesbaden 2004, S. 8-9.

18 Vgl. Mietzel, Gerd: Pädagogische Psychologie des Lernens und Lehrens. 8. Aufl. Göttingen 2007, hier S. 35-36.

$19 \mathrm{Vgl}$. Riedl (Anm. 17) hier S. 38-40 und 47-48.

${ }^{20}$ Vgl. Nilges, Annemarie: Informationskompetenz zwischen Strategie und Realität - Erfahrungen aus Nordrhein-Westfalen. In: Wissen bewegen - Bibliotheken in der Informationsgesellschaft. Frankfurt a. M. 2009. (Zeitschrift für Bibliothekswesen und Bibliographie; Sonderband 96) S. 81-92. 
von PCs, möglichst in einem eigenen Schulungsraum, verfügbar zu haben (Vgl. hierzu auch Kap. 4.2).

Generell lässt sich jedoch festhalten, dass sich kombinierte Methoden, die sowohl aktive als auch rezeptive Elemente für die Teilnehmer enthalten, bewährt haben und in vielen Bibliotheken Anwendung finden. So können sich an einen einleitenden Lehrvortrag oder eine Präsentation durch den Lehrenden Übungsaufgaben für die Teilnehmer anschließen, die in Einzel- oder Gruppenarbeit durchgeführt werden und in denen der theoretisch vermittelte Lernschritt praktisch nachvollzogen wird ${ }^{21}$.

Um den Lernfortschritt der Teilnehmer zu erfassen, bietet es sich zudem an, die Studierenden die Ergebnisse ihrer Übungsaufgaben im Anschluss im Plenum präsentieren zu lassen. Diese Methode, bei der eine Instruktions- und eine Präsentations- / Diskussionsphase eine Phase aktiver Erprobung „einrahmen“, wird auch als „Sandwichprinzip" bezeichnet ${ }^{22}$. Vorteilhaft bei dieser Methode ist zum einen die Abfolge verschiedener Phasen im Lernprozess, die einen Aufmerksamkeitsverlust, wie er z. B. bei reinen Lehrvorträgen oft auftritt, weitgehend vermeidet. In der Präsentationsphase können verschiedene Lösungswege diskutiert, Fehler konstruktiv genutzt werden. Die Bibliothek lernt die Denkweise der Studierenden und damit das Nutzerverhalten ihrer Hauptzielgruppe besser kennen. Diese Erkenntnisse können nicht nur zur Verbesserung der bibliothekarischen Lernangebote genutzt werden, sondern auch zur Optimierung der Funktionalität von Recherchesystemen beitragen.

Zudem werden die rhetorischen (während der Ergebnispräsentation) und kommunikativen (während der Diskussion) Fähigkeiten der Studierenden trainiert, was innen sowohl für das weitere Studium als auch in späteren beruflichen Situationen zugute kommen wird.

Bewährt hat sich in NRW auch eine Methode, die als „exploratives" oder „forschendes Lernen“ bezeichnet werden kann. Der Lehrende vermeidet in einer solchen Veranstaltung, den Stoff vorgefertigt vorzutragen. Stattdessen erschließen sich die Lernenden die Lerninhalte selbstständig, wobei innen die Vorgehensweise weitgehend freigestellt wird. Der Lehrende hält lediglich eine kurze Einführung, gibt dann Übungsaufgaben aus, die in Kleingruppen bearbeitet werden und steht im weiteren Verlauf der Veranstaltung für Fragen zur Verfügung. Anschließend werden die Übungsergebnisse von den Teilnehmern präsentiert und im Plenum besprochen ${ }^{23}$.

Auch wenn einige Bibliotheken Rundgänge durch ihre Räumlichkeiten oder Einführungen in die Katalogbenutzung zu Semesterbeginn wegen zu geringer Nachfrage aus ihrem Programm gestrichen haben, bedeutet das nicht, dass diese Angebote generell ihre Berechtigung verloren haben. Gerade die Bibliotheksführung, vor allem wenn sie im Rahmen von Erstsemestertagen zusammen mit Fachschaft oder Tutoren organisiert wird, kann den wichtigen Erstkontakt von Studienanfängern zur Bibliothek herstellen. Hier muss die Bibliothek unter Umständen ihre Ansprüche herunterschrauben und sich zunächst damit zufrieden geben, dass möglichst viele Erstsemester von der Existenz der Bibliothek erfahren, den Weg dorthin finden und mit einem Bibliotheksausweis ausgestattet werden. Das mag sich banal bis frustrierend anhören, ist aber in Zeiten zunehmender Nutzung des Internets zur Informationsrecherche und falscher Vorstellungen über dieses Medium traurige Realität an vielen Hochschulen.

E-Learning-Elemente kommen, in der einen oder anderen Form, in vielen Hochschulbibliotheken in NRW zum
Einsatz. Die Palette reicht dabei von statischen Selbstlernmaterialen, die in Form von aufbereiteten Dokumenten auf der Bibliotheks-Website zum Download angeboten werden, über Videosequenzen und animierten Selbstlernübungen zu einzelnen Themenbereichen, bis hin zum Angebot eigener E-Learning-Module auf der Lernplattform der Hochschule. Viele Bibliotheken nutzen zudem Online-Tutorials - entweder das von der AG Informationskompetenz konzipierte „Online-Tutorial Informationskompetenz" oder Eigenentwicklungen - zur Unterstützung ihrer Präsenzveranstaltungen.

E-Learning-Angebote können veranstaltungsbegleitend, zur Vor- bzw. Nachbereitung oder ergänzend, im Rahmen eines Blended Learning eingesetzt werden. Als Ersatz für Präsenzveranstaltungen sind sie aber keinesfalls ausreichend. Diese Erfahrungen teilen alle innerhalb der AG Informationskompetenz beteiligten Bibliotheken.

E-Learning kann außerdem als niedrigschwelliges Angebot für Studierende fungieren, die man mit Präsenzveranstaltungen nicht oder nur schwer erreicht. Dazu gehören $z$. B. kontaktscheue, technikaffine oder berufstätige Studierende.

\subsection{Weitere Tipps für die Praxis}

Wie bereits deutlich wurde, lassen sich didaktische Anforderungen in der Praxis nicht immer optimal umsetzen. Knappe Ressourcen bei gleichzeitig steigenden Teilnehmerzahlen machen es gelegentlich unvermeidlich, von lernorientierten Methoden abzurücken und zum klassischen Lehrvortrag zurückzukehren. Um diesen ansprechend zu gestalten, ist es sinnvoll, auflockernde Elemente zu integrieren und im Stil zu variieren. Dies kann zum Beispiel geschehen, indem der Vortrag stellenweise als Lehrgespräch geführt wird, der Vortragende Fragen stellt, zur Diskussion anregt und die Teilnehmer auf verschiedene Weise in den Ablauf einbezieht. Auflockernd wirkt auch der Einsatz von Medien (eine Veranstaltung im Hörsaal kann z. B. von Fotos der Bibliothek begleitet werden) oder die Gestaltung des Vortrags durch zwei Personen, die sich abwechseln. Generell ist bei der Methode Lehrvortrag zu empfehlen, langsam und deutlich zu sprechen, den Vortrag klar zu strukturieren, sich inhaltlich auf wesentliche Kernpunkte zu konzentrieren (didaktische Reduktion), diese gegebenenfalls zu visualisieren und immer wieder auch einmal Pausen einzulegen, in denen Raum für Fragen und Diskussion gegeben wird.

${ }^{21}$ Vgl. hierzu auch Sühl-Strohmenger, Wilfried: Neugier, Zweifel, Lehren, Lernen ...? Anmerkungen zur Didaktik der Teaching Library. In: Bibliotheksdienst 42 (2008) 8/9, S. 880-889.

22 Vgl. Rockenbach, Susanne: Teaching library in der Praxis Bedingungen und Chancen. In: Bibliotheksdienst 37 (2003) 1, S. 33-40. Lux, Claudia und Wilfried Sühl-Strohmenger: Teaching Library in Deutschland (B.I.T online: Innovativ; 9). Wiesbaden 2004, S. 46.

${ }^{23} \mathrm{Vgl}$. zu dieser Methode Rockenbach, Susanne: Neugier und Zweifel - Informationskompetenz anders. Vortrag, gehalten auf dem int. Kongress „Die lernende Bibliothek / La bibliotheca apprende", 2007 in Innsbruck <https://kobra.bibliothek. uni-kassel.de/handle/urn:nbn:de:hebis:34-2007100519309> [Abruf am 12.1.2009]. 
Eine Alternative zu dem input-orientierten Lehrvortrag ist die bereits angesprochene explorative Lernmethode. Sie eignet sich nicht nur - wie vielleicht anzunehmen für kleine Teilnehmerkreise, sondern durchaus auch für große Gruppen, vorausgesetzt, ein entsprechender Schulungsraum steht zur Verfügung. Neben den didaktischen Vorteilen für den Lernenden, der hier in hoher Weise eigenständig arbeiten kann, hat die Methode auch Vorteile für den Lehrenden. Sind die Übungsaufgaben erst einmal erstellt, laufen die Veranstaltungen fast von allein, sie sind also kraft- und ressourcenschonend. Der eigentliche Arbeitsaufwand besteht in der Konzeption der Übungsaufgaben. Hier ist es wichtig, sich vorab genaue Gedanken über die angestrebten Lernziele zu machen. Dabei kann man sich von Fachleuten aus der Hochschuldidaktik unterstützen lassen, die zu Beratungen gerne auch in die Bibliothek kommen ${ }^{24}$

Essentiell für Schulungen sind gute Kontakte in die Fakultäten bzw. Fachbereiche, insbesondere wenn es um die Beteiligung an regulären Lehrveranstaltungen der Hochschule geht. Oft hilft ein Blick in das Vorlesungsverzeichnis, um herauszufinden, welche Dozenten passende Veranstaltungen, wie z. B. fachliche Einführungen oder Grundlagen des wissenschaftlichen Arbeitens, abhalten. Auch Dekane oder Bibliotheksbeauftragte sowie nicht zuletzt die Fachschaften können dabei helfen, Kontakte herzustellen. Wurde ein Dozent für die Zusammenarbeit gewonnen, sollten auf Grundlage eines vorgefertigten Konzepts die notwendigen Rahmenbedingungen und Inhalte abgestimmt werden.

Das gute Gelingen einer Schulungsveranstaltung hängt jedoch nicht nur von Inhalt und Methodik, sondern auch von den organisatorischen Rahmenbedingungen ab.

\section{Informationskompetenz in der Bibliotheksorga-} nisation ${ }^{25}$

\subsection{Personalbedarf und Geschäftsverteilung}

Die Umsetzung von Vermittlung von Informationskompetenz ist in erster Linie abhängig vom Schulungskonzept der jeweiligen Bibliothek. Hat eine Bibliothek sich dafür entschieden, ihre Veranstaltungen selbstständig und "frei“ anzubieten, so kann sie auch Termine und Ort bestimmen und so erfahrungsgemäß die Zeiten, die im Bereich Erwerbung und Katalogisierung besonders arbeitsintensiv sind (Rechnungsabschluss etc.) aussparen. Ist man jedoch mit seinem Angebot in den Lehrbetrieb der Hochschule integriert, so muss man die Termine wahrnehmen, die von dort vorgegeben werden. Häufig hat man dann zu Beginn des Wintersemesters sehr viele Veranstaltungen zu übernehmen, im Sommer hingegen kaum Lehrverpflichtungen. Diese ruhigeren Sommermonate können jedoch für die Bibliotheken, die eigenständige Veranstaltungen anbieten, auch eine Möglichkeit sein, ihre Seminare als Blockkurse außerhalb des normalen Lehrbetriebs anzubieten. Das wird von Studierenden in der Regel sehr gerne angenommen, da diese oft während der Semester kaum Zeit für weitere Veranstaltungen haben.

Viele Bibliotheken leisten sich nach wie vor zwei unterschiedliche Schulungsprogramme: ein fakultatives oder „freies“, d. h. nicht in die Lehre integriertes Programm, das von den erfahrenen Diplom-Bibliothekarinnen und -Bibliothekaren durchgeführt wird, und ein fachspezifisches, das in der Verantwortung der Fachreferate bzw. von Fachteams liegt. Je weiter jedoch die Anbindung an die Fach-Curricula fortgeschritten ist und damit auch die Zahl der Veranstaltungen und vor allem die Zahl der Teilnehmer deutlich steigt, desto notwendiger wird eine institutionalisierte Kooperation aller an Schulungen beteiligten Kräfte des Hauses. Sehr schnell kann es notwendig werden, Tutoren oder Fach-Dozentinnen und Dozenten als Multiplikatoren für Informationskompetenz einzusetzen, da das bibliothekarische Personal rein quantitativ nur selten in der Lage sein wird, alle Studierenden ausreichend zu schulen. Bei semesterlangen Veranstaltungen der Bibliothek beantragt in der Regel ein Fachreferent einen Lehrauftrag der Fakultät, in der das Seminar angesiedelt wird. Damit hat er oder sie die Verantwortung für den Kurs auch gegenüber dem Prüfungsamt, wenn ECTS vergeben werden. Die einzelnen Sitzungen des Seminars können jedoch auf mehrere Schultern verteilt werden. Lokale Bibliotheksbenutzung, Online-Katalog sowie Fernleihe und Dokumentlieferung sind klassische fachübergreifende Themen, für die es in der Regel in jeder Bibliothek Spezialisten aus dem gehobenen Dienst gibt, die normalerweise gerne bereit sind, diese Sitzungen auch im Rahmen eines Seminars zu übernehmen. Ebenso lassen sich mehrere ähnliche Fächer wie die Philologien oder sogar alle mathematisch-naturwissenschaftlichen Fächer oder die Sozialwissenschaften personaleffizient zu Seminaren zusammenfassen, an denen dann jeweils auch unterschiedliche Fachreferenten die fachspezifischen Angebote der einzelnen beteiligten Fächer schulen. Grundsätzlich, vor allem jedoch für die üblichen 90-minütigen Veranstaltungen (Bibliothekseinführung, Online-Katalog und ausgewählte Datenbanken), egal ob integriert oder frei angeboten, Schülerführungen etc., ist der Personaleinsatz am effizientesten zu gestalten, wenn man ein festes „Team IK“ bildet, dessen Mitglieder zu einem bestimmten Teil ihrer Arbeitszeit die Vermittlung von Informationskompetenz als Kernaufgabe haben. Dieses Team sollte bei Bedarf (Urlaub, Krankheit, besonders schulungsintensive Zeiten) durch zusätzliche Mitarbeiterinnen und Mitarbeiter aus dem Haus ergänzt werden können. Die Mitglieder treffen sich regelmäßig zum Austausch, erarbeiten gemeinsame Schulungsunterlagen und organisieren Fortbildungen und Vertretungen. Dem Kern-Team obliegt auch das Marketing für die eigenen Veranstaltungen ${ }^{26}$. Zusätzlich nehmen die Mitglieder die wichtige Funktion von Ansprechpartnern für die Lehre bzw. für Nachfragen zu Schulungsterminen wahr. Denkbar ist vor allem in großen Bibliotheken eine Arbeitsaufteilung innerhalb des Teams. Marketing, das Erarbeiten von Unterlagen, der Auftritt auf der Homepage und der Lernplattform oder das Filtern der Angebote im Fortbildungsbereich auf für die eigene Bibliothek interessante Seminare hin können Aufgaben sein, für die einzelne Mitglieder

${ }^{24}$ Weitere Informationen, Anregungen, Materialien zu Lerninhalten, Methoden u. Ä. finden sich in der Materialiendatenbank des Portals <http://www.informationskompetenz.de>.

25 Die Ausführungen haben die verschiedenen Standards zur Vermittlung von Informationskompetenz zur Grundlage. Vgl. Nilges, Annemarie; Reessing-Fidorra, Marianne und Renate Vogt: Standards für die Vermittlung von Informationskompetenz an der Hochschule. In: Bibliotheksdienst 37 (2003) 4, S. 463-465.

${ }^{26}$ Für die in das Lehrangebot integrierten Veranstaltungen ist es normalerweise nicht notwendig, Marketing zu betreiben. 
des Teams verantwortlich zeichnen. Zum Kernteam gehören in der Regel die Fachreferentinnen und Fachreferenten, soweit sie schulen, und Diplom-Bibliothekarinnen und Diplom-Bibliothekare aus dem Auskunfts- oder Informationsbereich. Zusätzliches Personal kann gerade aus den Abteilungen ohne direkten Benutzerkontakt wie Medienbearbeitung etc. kommen, da die Kollegen dort häufig froh sind, der Schreibtischroutine für einige Stunden zu entkommen. Wichtig ist jedoch, dass alle, sowohl Mitglieder des Kernteams als auch zusätzliche Kräfte ausreichend auf ihre Aufgaben vorbereitet sind, einen vergleichbaren Stand in Didaktik, Methodik etc. haben und über ausreichend Zeit für die Vor- und Nachbereitung ihrer Veranstaltungen verfügen.

Als Faustregel bei der Berechnung des Arbeitsanfalls für eine Veranstaltung - und damit grundsätzlich auch zur Berechnung der notwendigen Personalkapazität für das Gesamtkonzept - hat es sich bewährt, die Dauer der Veranstaltung mal zwei zu nehmen ${ }^{27}$. Das kann weniger sein, wenn der Schulungsbibliothekar sehr routiniert ist und es sich um Veranstaltungen handelt, die er oder sie schon oft durchgeführt haben. In diesem Fall ist nur kurz vor dem Termin zu klären, ob die Beispiele noch aktuell sind bzw. die Oberflächen der Datenbanken und sonstigen Systeme sich nicht geändert haben. Hat man natürlich das didaktische Konzept umgestellt, will etwas Neues probieren oder übernimmt man eine Veranstaltung, die einem inhaltlich neu ist, so kann die Vorbereitungszeit erheblich darüber liegen. Auch die Erarbeitung einer semesterlangen Veranstaltung ist sehr arbeitsintensiv, weswegen man hier wirklich Kontinuität anstreben und eine derartige Veranstaltung möglichst mehrfach, z. B. mehrere Semester in Folge, anbieten sollte. Wichtig bei der Berechnung der Zeiten und damit des Personaleinsatzplanes ist ebenfalls, ob die Schulungsbibliothekare zusätzlich noch für die Organisation ihrer Schulungsräume verantwortlich sind. Es ist äußerst zeitintensiv, wenn der Schulende sich vorab vergewissern muss, in welchem Zustand der Raum gerade ist - inklusive etwaiger Aufräum- oder Umräumarbeiten - und zusätzlich noch PC und Beamer am Lehrer-PC und eventuell auch noch an den BenutzerPCs hochfahren und deren Funktionieren selbst überprüfen muss. Die dafür notwendige Zeit muss unbedingt der oben angegebenen durchschnittlichen Vorbereitungszeit hinzugerechnet werden. Idealerweise, jedoch in der Praxis leider äußerst selten, verfügt die Bibliothek über ausreichendes technisches Personal, das einem diese Arbeiten abnimmt, so dass der Schulungsbibliothekar sich auf Inhalte und Didaktik konzentrieren kann. Nicht zu unterschätzen ist auch die Nachbereitung einer Veranstaltung. Abgesehen von den organisatorischen Dingen - Herunterfahren der Geräte, Aufräumen des Raumes, falls es dafür kein anderes Personal gibt - besteht auch oft noch Gesprächsbedarf aus der Veranstaltung heraus. Oft kommen noch Teilnehmer mit Spezialfragen oder Verständnisfragen. Je größer die Gruppe war, desto häufiger kam es vor, dass Teilnehmer sich nicht getraut haben, sich vor der großen Gruppe zu äußern und lieber im Nachhinein das Gespräch suchten. Sehr wichtig auch aus Evaluationsgründen und unter Marketinggesichtspunkten ist der anschließende kurze Austausch mit dem Dozenten, um festzustellen, ob die Erwartungen erfüllt wurden, ob man die Schwerpunkte hätte anders setzen sollen etc. Hat kein Dozent teilgenommen, sondern ist die Gruppe selbständig in der Bibliothek erschienen, empfiehlt sich trotzdem eine kurze Kontaktaufnahme mit dem Dozen- ten nach der nächsten Sitzung des Kurses etwa per Mail oder telefonisch, um zu erfahren, wie die Teilnehmer sich noch zu der Sitzung geäußert haben oder sogar Lernerfolge festzustellen sind etc. Hierdurch setzt man auch für die Studierenden ein deutliches Zeichen, dass die Bibliotheksveranstaltungen in die Lehre integriert sind. Unbedingt sollte man nach einer Veranstaltung eine kurze Ruhepause oder Phase mit leichteren Routineaufgaben einplanen, da die Gestaltung einer Schulungsveranstaltung den Lehrenden normalerweise sehr fordert.

Tabelle 2: Checkliste der vor und nach einer Schulungsveranstaltung zu erledigenden Dinge

\begin{tabular}{|c|c|}
\hline \multicolumn{2}{|c|}{ Eingangsdiagnostik: } \\
\hline & - Fach/Titel des Kurses \\
\hline & - Dozent \\
\hline & - Teilnehmeranzahl \\
\hline & - Niveau der Zielgruppe \\
\hline & $\begin{array}{l}\text { - Besondere Wünsche seitens des Dozenten an die } \\
\text { Inhalte der Veranstaltung }\end{array}$ \\
\hline \multicolumn{2}{|c|}{ Inhaltliche Planung: } \\
\hline & - Inhalte festlegen \\
\hline & $\begin{array}{l}\text { - Didaktik/Methodik überlegen (Gruppengröße und Ni- } \\
\text { veau berücksichtigen) }\end{array}$ \\
\hline & $\begin{array}{l}\text { - Materialien auf Aktualität hin überprüfen, überprüfen } \\
\text { ob Oberfläche von Datenbanken noch wie beim letz- } \\
\text { ten Mal }\end{array}$ \\
\hline & - Beispiele ausprobieren \\
\hline & - Handout notwendig \\
\hline & $\begin{array}{l}\text { - Unterlagen für den Semesterapparat oder die Lern- } \\
\text { plattform gewünscht }\end{array}$ \\
\hline \multicolumn{2}{|c|}{ Organisation: } \\
\hline & - Raum buchen (Größe der Gruppe beachten) \\
\hline & $\begin{array}{l}\text { - Methodik/Didaktik festlegen, - Raumausstattung } \\
\text { daraufhin überprüfen (Beamer, PCs notwendig, ja oder } \\
\text { nein, Tische für Gruppenarbeit, Tafel, Flipchart etc.) }\end{array}$ \\
\hline & $\begin{array}{l}\text { - Pförtner/elektronisches Leitsystem etc. informieren, } \\
\text { um die Gruppe zu empfangen }\end{array}$ \\
\hline & - Evtl. für Vertretung bei den Kernaufgaben sorgen \\
\hline & $\begin{array}{l}\text { - Kurz vor der Veranstaltung den Raum und Ausstat- } \\
\text { tung überprüfen/herrichten }\end{array}$ \\
\hline \multicolumn{2}{|c|}{ Evaluation: } \\
\hline & $\begin{array}{l}\text { - Feedback am Ende der Sitzung organisieren (Punkte } \\
\text { kleben, Kritikgespräch, Fragebogen, (nur bei mehr- } \\
\text { teiligen Veranstaltungen oder solchen von mehr als } \\
90 \text { Min. Dauer empfehlenswert) }\end{array}$ \\
\hline & $\begin{array}{l}\text { - Unterlagen für die Sitzung für die Wiederverwendung } \\
\text { abheften }\end{array}$ \\
\hline & $\begin{array}{l}\text { - Veranstaltung für die statistische Auswertung notie- } \\
\text { ren }\end{array}$ \\
\hline & $\begin{array}{l}\text { - Pförtnern, Kollegen, die vertreten haben, EDV, Raum- } \\
\text { service etc. danken }\end{array}$ \\
\hline & $\begin{array}{l}\text { - Ca. eine Woche bis zehn Tage nach der Veranstaltung } \\
\text { Dozenten kontaktieren und fragen, ob es ein Feedback } \\
\text { der Gruppe gab, er/sie noch Änderungswünsche hat, } \\
\text { was sein/ihr Gesamteindruck war... }\end{array}$ \\
\hline
\end{tabular}

In jeder Bibliothek sollte es einen Beauftragten für Informationskompetenz geben. Häufig besetzt er oder sie eine ${ }_{27}$ Vgl. hierzu das Ausbildungskonzept zur studienbegleitenden Vermittlung von Informationskompetenz der Universitäts- und Landesbibliothek Düsseldorf unter <http://www. ub.uni-duesseldorf.de/docs/konzept_ik.pdf> [Zugriff am 15.4.2009]. 
entsprechende Stabsstelle, die im Bereich der Direktion oder innerhalb eines Dezernats - in Nordrhein-Westfalen üblicherweise im Dezernat Benutzung - angesiedelt ist. In jedem Fall handelt es sich um eine Querschnittsaufgabe über alle Dezernate hinweg, und entsprechend sollte auch das Profil der Stelle gestaltet werden. Es muss dem Informationskompetenz-Beauftragten möglich sein, Personal aus unterschiedlichen Dezernaten anzuwerben, fortzubilden und einzusetzen, natürlich jeweils unter Beachtung der Kernaufgaben der Kolleginnen und Kollegen. Sicherlich kann man niemand aus der Zeitschriftenabteilung in der Zeit um den Rechnungsschluss intensiv mit der Wahrnehmung von Schülerveranstaltungen betrauen. Die Stabsstelle IK sollte auch einen kurzen Weg zur Direktion haben, dort jederzeit gehört werden und auch von der Direktion über alle den Lernort Bibliothek betreffenden Projekte und Planungen unterrichtet bzw. in diese miteinbezogen werden.

\subsection{Lernort Bibliothek: Anforderungen an Räume und Ausstattung}

Organisatorisch sollte der Lernort Bibliothek grundsätzlich so gestaltet werden, dass Lernen als integraler Bestandteil des täglichen Lebens verstanden wird und sowohl am stationären PC als auch mit dem mitgebrachten Notebook überall individuell oder in verschiedenen Gruppenarbeitskonstellationen möglich ist ${ }^{28}$. Ebenso sind geeignete Räumlichkeiten für Blended Learning-Szenarien in größeren oder kleineren Schulungsgruppen vorzusehen, denn immer mehr Veranstaltungen enthalten Einheiten zum eigenen selbst gesteuerten Lernen z. B. über OnlineTutorials. Auch ein Atrium, eine Lounge, Lern-Café und / oder Entspannungsbereiche für kommunikative Begegnungen mit den Mit-Lernenden, ausgestattet mit entsprechend ansprechendem Mobiliar, sind wünschenswert ${ }^{29}$. Hier bieten die zusätzlichen Mittel aus den Studienbeiträgen, sofern Platz vorhanden ist, gute Möglichkeiten, in Zusammenarbeit mit den Studierenden / Lernenden geeignete Räume zu schaffen bzw. auszugestalten.

Bei der Konzeption von Schulungsräumen ist Flexibilität der oberste Maßstab. Nach wie vor gilt grundsätzlich, wie bereits 2003 von der Arbeitsgruppe Informationskompetenz des Landes Nordrhein-Westfalen formuliert ${ }^{30}$ :

Die Schulungsräume

- befinden sich im Idealfall in der Bibliothek, oder sie stehen der Bibliothek regelmäßig für Schulungsveranstaltungen zur Verfügung,

- erfüllen die unterschiedlichen Anforderungen für Einzeloder Kleingruppenberatung, multimediale Schulungen für Gruppen bis zu 20 Personen, für Präsentationen bzw. Vorträge für Gruppen von 20-100 Personen,

- entsprechen bezüglich der Lichtverhältnisse, der Ergonomie des Mobiliars und der klimatischen Bedingungen den an Schulungsräume üblicherweise zu stellenden Anforderungen,

- verfügen über eine Ausstattung nach den Maßstäben der Technology for Training (TFT): Tafel, Flip-Chart, Overheadprojektor und PC mit Beamer, außerdem im multimedialen Schulungsraum (mindestens) zehn PCs, im Vortragsraum eine Mikrofonanlage,

- sind hinsichtlich der Geräteausstattung und der Anordnung des Mobiliars multifunktional zu nutzen,
- sind leicht zu buchen und die Belegung ist transparent (z. B. über eine im Intranet aufliegende Datenbank mit folgenden Informationen: Termin, Dauer der Veranstaltung, Titel der Veranstaltung, Veranstalter, Dozent, gewünschter Raum und gegebenenfalls welche Zusatzausstattung).

Da kaum eine Bibliothek über unterschiedliche Schulungsräume für alle denkbaren und in der Schulungspraxis gängigen didaktischen Szenarien verfügt, ist besonders die Multifunktionalität wichtig. Hat die Bibliothek nur einen Raum zur Verfügung, so sollte dieser neben einem Lehrer-PC und einem Beamer so viele PCs wie möglich enthalten. Da der Raum dann auch dazu dient, kleine Impulsreferate zu halten oder eine Präsentation voran- oder hintanzustellen und vermutlich nicht die ganze Sitzung über am PC geübt wird, ist es wichtig zu gewährleisten, dass die Teilnehmer nicht in Versuchung geführt werden, statt zuzuhören, im Internet zu surfen etc. Der Raum sollte auch die Möglichkeit zur Gruppenarbeit bieten, d. h. idealerweise ist ein Teil der Tische beweglich und kann je nach Lernsituation neu arrangiert werden.

Bibliotheken, die sich entscheiden, unabhängig vom Lehrbetrieb ihre Schulungsveranstaltungen in eigener Regie anzubieten, werden vermutlich mit einem einzigen, dafür aber multifunktionalen Raum auskommen, da sie die Gruppengröße selbst festlegen und in der Regel nicht mehr als 20 bis 30 Teilnehmer annehmen werden.

Sieht das Konzept der Bibliothek die Integration in die Curricula vor und werden eigene semesterlange Veranstaltungen durchgeführt, ist von mittelgroßen bis großen Gruppen auszugehen. Gerade geisteswissenschaftliche Seminare umfassen heute in der Regel zwischen 50 und 100 Teilnehmer. Wenn man diese, wie didaktisch unbedingt angeraten, nicht nur im Vorlesungsstil unterrichten sondern auch aktivieren möchte, so sollte der Schulungsraum über so viele PCs verfügen, dass sich, wenn man die Gruppe geteilt hat, je zwei Studierende ein Gerät teilen können. Hat der Raum nur zehn PCs und ist es auch nicht möglich, einen Raum in der Fakultät oder im Rechen-

${ }^{28}$ Aus der neueren Literatur vgl. Fansa, Jonas: Bibliotheksflirt: Bibliothek als öffentlicher Raum. Bad Honnef 2008. Beiser, Sylvia: Lernort Bibliothek. In: B.I.T. online 11 (2008) 4, S. 431-437. Bisbrouck, Marie-Francoise (Hrsg.): Libraries as places: Buildings for the $21^{\text {st }}$ century (ILFA-Publications; 109). München 2004. Dies. (Hrsg.): Library Buildings in a Changing Environment (IFLA-Publictions; 94) München 2001. Dies. (Hrsg.): Intelligent Library Buildings (ILFA-Publications; 88) München 1999. European Research Libraries Cooperation /Architecture Group (Hrsg.): The postmodern library between functionality and aethetics. Graz 1997. Bd. 1 mit Schwerpunkt Teaching Library.

${ }^{29}$ Vgl. Z. B. Higher Education Funding Council for England (HEFCE): Designing Spaces for Effective Learning: A guide to $21^{\text {st }}$ century learning space design. $2006<$ http://www.jisc. ac.uk/uploaded_documents/JISClearningspaces.pdf $>$ [Zugriff am 15.4.2009]. École Polytechnique Fédérale de Lausanne: Some reflexions to build a learning center <http:// learningcenter.epfl.ch/page56165.html> [Zugriff am 15.4.2009]. Latimer, Karen und Hellen Niegaard (Hrsg.): IFLA Library Building Guidelines: Developments \& Reflections. München 2007.

${ }^{30}$ Nilges/Reessing-Fidorra/Vogt (Anm. 25), hier S. 464. 
zentrum zu bekommen, so ist das Problem didaktisch anzugehen, indem man die Studierenden mit gezielten Aufgaben in den Lernort Bibliothek entlässt und hinterher im Plenum die Aufgaben bespricht. Alternativ könnte man die zur Verfügung stehende Zeit kontingentieren und jeweils unterschiedliche Gruppen für eine bestimmte Zeit üben lassen, während der Rest andere Aufgaben bearbeitet. Hat man häufig größere Gruppen, so lohnt es sich in jedem Fall, neben dem Raum für praktische Übungen am PC auch einen Vortragsraum zu haben, der z. B. auch für Ausstellungseröffnungen, Kongresse oder sonstige Veranstaltungen der Lehrstühle genutzt werden kann. Wenn man die Räume über ein transparentes Buchungssystem auch der universitären Öffentlichkeit zur Verfügung stellt, ist es häufiger möglich, die notwendige Unterstützung in der Hochschule für die Einrichtung derartiger Räumlichkeiten zu finden.

\subsection{Marketing: Informationskompetenz als Entrée in die Hochschule}

Viele Bibliotheken, die ihre Veranstaltungen nicht in die reguläre Lehre einbringen (können), leiden darunter, dass eigentlich viel zu wenig Nutzer das Schulungsangebot wahrnehmen und sie so, betriebswirtschaftlich gesehen, eine sehr ungünstige Kosten/Personalaufwand/NutzenRelation haben. Hier kommt der Werbung eine wichtige Bedeutung zu. Sie sollte zielgruppenspezifisch sein. Plakate, Flyer, Serienbriefe, E-Mails, Newsletter, Werbung über die Homepage, Durchsagen, Presseartikel etc. sollten sich in Sprache und Inhalt an der jeweils angesprochenen Zielgruppe orientieren. Auch die Zusammenarbeit mit hochschulinternen oder externen Gremien hat sich bewährt. Erstsemester erreicht man z. B. über die von den Studierendenorganisationen initiierte Werbung, wie sie z. B. in den zu Beginn des Wintersemesters verteilten „Ersties-Tüten“ zu finden ist. Oder über das Campus-Radio oder die Campus-Zeitung. Je integrierter das Schulungsangebot einer Bibliothek ist, desto weniger muss sie ihre Veranstaltungen bewerben. Streng genommen kann sogar der Hinweis darauf auf der Homepage der Bibliothek entfallen, da die Studierenden die Kurse ohnehin über das Online-Vorlesungsverzeichnis buchen und sich ihre Informationen dort besorgen. Hier hat die Werbung vornehmlich noch den Zweck der Selbstdarstellung der Bibliothek innerhalb der Hochschule oder den Sponsoren und anderen Unterhaltsträgern gegenüber.

Da das Schulungsangebot der Bibliothek vor allem bei den fachübergreifenden Wahlpflichtveranstaltungen eine echte Bereicherung des Angebots der Hochschule darstellt und von den meisten Hochschulen daher sehr geschätzt ist, kann ein Engagement der Bibliothek bei der Vermittlung von Informationskompetenz auch Türen in der Hochschule öffnen, die sonst schnell verschlossen bleiben, weil die Relevanz bibliothekarischer Themen für Forschung und Lehre nicht so deutlich ist. Gerade in diesem Zusammenhang ist es wichtig, sich dessen bewusst zu sein, dass Schulungsveranstaltungen nur eine Facette der Vermittlung von Informationskompetenz sind, und dass diese eigentlich viel mehr ist, dass sie nämlich als Schlüsselqualifikation durchaus zur Profilbildung für gesamte bibliothekarische und informationswissenschaftliche Angebote herangezogen werden kann.
Nicht zuletzt aus diesem Grund sind Qualitätssicherung und Evaluierung wichtig.

\section{$5 \quad$ Evaluation}

\subsection{Einführung}

Das Wörterbuch des Qualitätsmanagements definiert Evaluation, vielmehr „Evaluierung“, als „Prozess zum Bewerten der Erfüllung der Anforderungen des Zertifizierungsprogramms durch eine Person, der zur Entscheidung zur Zertifizierung führt" 31 . Diese stark formalisierte Definition gehört in den Kontext durchdeklinierter Qualitätsmanagementverfahren. Einfacher ausgedrückt bedeutet Evaluation schlicht „Bewertung“. Es gibt eine Reihe sehr verschiedener Methoden der Leistungs- oder Erfolgsmessung. Ausschlaggebend ist, welches Erkenntnisinteresse und damit welcher Gegenstand ihr zugrunde liegt. Davon abhängig verwendet man unterschiedliche Methoden der Datenerhebung, Datenanalyse und Ergebnisdarstellung. In der Regel soll die Evaluation zur Anpassung oder Optimierung eines Leistungssystems führen oder das Erreichen bestimmter Ziele überprüfen. Ein zu erreichendes Ziel bei Schulungsangeboten könnte z. B. die Marktdurchdringung innerhalb einer Klientel sein oder aber die Einstellung eines Schulungsangebotes aufgrund hochwertiger Katalogsysteme oder User-Interfaces bzw. aufgrund der verbesserten Informationskompetenz der Kunden. Wichtig ist, dass die Ziele klar definiert und geprüft werden und das Angebot durch die Organisation hindurch gelebt wird. Das Scheitern von Schulungsangeboten hat häufig seine Ursache nicht in der Durchführungsqualität einer einzelnen Veranstaltung, sondern darin, dass die Organisation Bibliothek nicht insgesamt beteiligt ist.

Evaluation liefert je nach Ausrichtung Daten für politische, strategische oder wirtschaftliche Ziele (Imageverbesserung, Marktplatzierung, Leistungsdarstellung vor dem Träger, Ressourcenverwendung), Planungen und Entscheidungen (Produktentwicklung, Optimierung von Prozessen) oder für ein Forschungsinteresse (Informationswissenschaft). Dementsprechend wird der Untersuchungsgegenstand gewählt (Nutzung, Image, Bekanntheitsgrad, Rechercheverhalten) und ein bestimmtes Erkenntnisinteresse (Fakten oder Beziehungen in Form von einzelnen Werten im Verhältnis zueinander, in Form von UrsacheWirkung oder von Korrelationen) formuliert. Unterschieden werden Input-Output-Relationen im Hinblick auf Effizienz (einfache Kosten-Nutzen-Analyse, z. B. Kosten einer Veranstaltung im Bezug zur Teilnehmeranzahl) oder kurzund mittelfristige Effektivität (Lernerfolg, Entwicklung der Nutzungszahlen, des Aktivierungsgrades von Beständen und Datenbanken, Marktdurchdringung) und Input-Outcome-Relationen $^{32}$. Hierzu gehören beispielsweise die Beziehung von Schulungen etc. zur Kundenbindung oder Kundenzufriedenheit sowie der „impact“, also langfristige

${ }^{31}$ Graebig, Klaus: Wörterbuch Qualitätsmanagement. Berlin 2006, S. 32.

32 Vgl. dazu Umlauf, Konrad: Leistungsmessung und Leistungsindikatoren für Bibliotheken im Kontext der Ziele von Nonprofit-Organisationen (Berliner Handreichungen zur Bibliothekswissenschaft; 116). Berlin 2004, S. $11 \mathrm{ff}$. 
Wirkungen von Maßnahmen auf das Informationsverhalten, den beruflichen Erfolg oder Haltungen von Personen. Die beiden wichtigen statistischen Erhebungsinstrumente im Bibliothekswesen, die Deutsche Bibliotheksstatistik $(\mathrm{DBS})^{33}$ und der Bibliotheksindex (BIX) ${ }^{34}$, zählen die Stunden, die jährlich für Schulungen investiert werden sowie die Zahl der Teilnehmer (DBS) bzw. die Schulungsstunden pro 1000 Nutzer aus der primären Nutzergruppe (BIX). Der BIX-Indikator drückt bereits eine Beziehung aus und misst im Grunde Nutzungsfrequenz und Marktdurchdringung bei einer bestimmten Klientel. Doch nicht nur die Dimension der Schulungszahlen selbst ist Indikator für den Erfolg oder die Wirkung, auch die Nutzung anderer Angebote, wie sie im BIX oder in der DBS aufgeführt werden (Nutzung von Datenbanken etc.), weist eventuell Korrelationen zum Faktor Schulungen auf. Die Interdependenzen müssen natürlich genauer aufgezeigt und nachgewiesen werden. Der genannte Indikator im BIX zu Schulungen sagt wenig über die Qualität einer Schulung im Sinne der Produktqualität oder im Sinne der Prozessqualität der Leistungserstellung aus.

Produkt- und Prozessqualität beeinflussen jedoch im erheblichen Maße die einfachen „messbaren“ Werte zur Effizienz: wenn ein schulungswilliger Kunde nie jemanden erreicht, der seine Anmeldung zur Schulung entgegennimmt, kann das sowohl die Kundenzufriedenheit als auch die Nutzung negativ beeinflussen. Die Anmeldungsorganisation, so profan einem das vorkommen mag, ist ein typisches Kriterium der Prozessqualität des Gesamtvorgangs Schulungen. Weiter würde z. B. eine Schulung, die keinerlei standardisierte Bestandteile hat und folglich immer neu organisiert werden muss, den Faktor Effizienz ebenfalls erheblich ins Negative ziehen. Es kann aber auch den gegenteiligen Fall geben, nämlich eine überdimensionierte Vorhaltung von Kapazitäten, obwohl die Kundschaft mit einfachen Lösungen hochwertig zufrieden zu stellen ist.

Input-Outcome-Analysen, z. B. der Nachweis langfristiger Wirkung von Angeboten auf das Nutzungsverhalten in Bibliothekssystemen oder für die Wertschöpfung durch den Kunden in verschiedenen Kontexten, bedürfen sehr aufwendiger sozial- bzw. informationswissenschaftlicher Verfahren. Insbesondere die Informations- und Bibliothekswissenschaft im angloamerikanischen Raum hat zum Thema „impact“ von Bibliotheksarbeit interessante Modelle und Studien vorgelegt ${ }^{35}$. Auswirkungen auf Informationsverhalten, Bildung, Kompetenzen der beruflichen oder Lebensgestaltung sind eigentlich die entscheidenden Kontexte bibliothekarischer Arbeit; davon sind wir Bibliothekare mit Recht überzeugt und das währt hoffentlich ein ganzes Berufsleben lang. Hier - in unserem intendiert praktisch angelegten Handlungsleitfaden - werden wir uns jedoch im Folgenden auf eher praktische Möglichkeiten der Evaluation von Informationskompetenz-Angeboten für den Bibliotheksalltag konzentrieren.

Die einzelnen Evaluationsformen werden auch als formativ oder summativ unterschieden. Formative Evaluationen sind eher auf einzelne messbare Ergebnisse aus, die die Qualität einer Maßnahme sichern sollen, z. B. ein Feedback nach einer Veranstaltung. Summative Erhebungen beziehen meist über längerfristige Untersuchungen Bilanz bezüglich der Wirkung von Maßnahmen. Die einzelnen Erhebungsmethoden sind in der Regel das statistische Zählen von Vorgängen etc., eventuell elektronisch ge- stützte Statistik oder die Methoden Beobachtung, Befragung, Interview, Test usw. Quantitative Datenerhebungen (Nutzungszahlen) stehen qualitativen Verfahren (Rechercheanalyse) gegenüber. Häufig wird versucht, qualitative Aspekte durch eine Gesamtbetrachtung mehrerer quantitativer Erhebungen oder die Analyse von Interdependenzen verschiedener Indikatoren abzubilden.

\subsection{Produktorientiertes Qualitätsmanagement - einzelne Evaluationsmöglichkeiten}

Es folgt eine Ordnung nach Erkenntnisinteresse / -gegenstand, die dem praktischen Ansatz entgegenkommt:

Nutzung des Schulungsangebots (Effizienz) im Sinne von Auslastung, Kosteneffizienz und Marktdurchdringung:

- Nutzungszahlen pro Veranstaltung.

- Schulungsstunden pro Jahr im Verhältnis zur Anzahl von (potentiellen) Nutzern einer bestimmten Gruppe (Primärgruppe, spezielle Nutzertypen wie Jugendliche etc.) zu bestimmten Zeiten oder in bestimmten Zweigstellen etc. Dies misst Nutzungsfrequenz und Marktdurchdringung, zeigt auch soziale, kulturelle Verhaltensformen auf.

- Erhebung tatsächlicher Kosten im Verhältnis zur Teilnehmerzahl oder im Verhältnis zu anderen Maßnahmen der Kundeninformation etc.

Diese Daten lassen sich relativ einfach erheben und sind häufig Bestandteil des Controllings von Hochschulen oder Kommunen. Eventuell gehen sie in einen Leistungsvergleich, ein Benchmarking ein. Wichtig im Sinne prozessualer Qualitätsbetrachtung ist, dass die Bibliothek dafür Erfassungsmittel entwickelt und Ressourcen bereithält (Erfassungsbögen, Datenbank, kompetente Bearbeiter/innen). Dies gilt grundsätzlich für alle Evaluationsformen.

Marktposition, das Image der Bibliothek (Effektivität 1) im Sinne von „Platzierung“ in einem Segment, das den Kernaufgaben entspricht oder mit dem man sich „profilieren" kann:

- Erhebung von Nutzungszahlen im Sinne der Marktdurchdringung (s. o.) bei Schulungen.

- Erhebung über den Bekanntheitsgrad und das öffentliche Erscheinungsbild bei Nutzergruppen, allen Nutzern, Nichtnutzern, Multiplikatoren, Medien.

- Beobachtung öffentlicher Resonanz: Auftreten von Anfragen bei der Bibliothek nach „Experten“ zum Thema Informationskompetenz (Kongresse, Schulungen), Entwicklung von Kooperationen, Förderung durch öffentliche Institutionen oder Sponsoren.

Lernerfolg (Effektivität 2) im Sinne von Erhöhung der Kompetenzen im Umgang mit Informationsmitteln:

${ }^{33}$ Deutsche Bibliotheksstatistik (DBS) <http://www.hbz-nrw. de/angebote/dbs/> [Zugriff am 26.11.2008].

${ }^{34}$ Bibliotheksindex (BIX) <http://www.hbz-nrw.de/angebote/ $\mathrm{dbs} /$ bix/> [Zugriff am 26.11.2008].

${ }^{35}$ Ein praktischer Überblick dazu findet sich in Markless, Sharon und David Streatfield: Evaluating the impact of your library. London 2006. 
- Einschätzung des Kunden zum Lernerfolg, z. B.: „Können Sie die Datenbank X benutzen?" „Wissen Sie, wie man die Funktion $X$ im Katalog benutzt?"

- Nutzung von Recherchemitteln vor und nach der Veranstaltung: wie oft wurden die vorgestellten Systeme vorher, wie oft nachher benutzt (quantitativ)?

- Rechercheverhalten bis hin zur vergleichenden Usability-Forschung: Datenbanken, Kataloge werden laufend auf ihre „usability“ hin geprüft (wie recherchieren Nutzer bzw. wo „bleiben sie hängen“). Dies dient dem Vergleich von Recherchestrategien vor und nach einer Schulung, aber auch einem Schulungskonzept (qualitativ).

- Anzahl und Art von Informationskompetenz-relevanten Fragen an Auskunftsplätzen und über elektronische Systeme: verändern sich die Fragen (qualitativ)?

- Prüfung von Kenntnissen (Test) anhand von Fragen zu Suchstrategien, Inhalt und Funktionen von Rechercheinstrumenten etc., eventuell über längere Testreihen Untersuchung der Nachhaltigkeit der Kenntnisse oder der Fähigkeit zum Transfer auf andere Systeme.

- Evaluation anhand von Standards für Informationskompetenz des Nutzers: Voraussetzung ist, dass die Standards zertifizierbar sind. Ein Standard muss so bestimmt formuliert sein, dass das Erreichen nachgeprüft werden kann.

Solche Standards sind z. B. die „Competency Standards for Higher Education" 36 der Association of College and Research Libraries (ACRL), für die seit 2002 eine deutsche Übersetzung vorliegt ${ }^{37}$. Modifikationen der Standards sind durch die AG Informationskompetenz BadenWürttemberg erarbeitet worden ${ }^{38}$. Die ACRL-Standards definieren Lernziele und Indikatoren, die das Erreichen des Zieles anzeigen, beispielsweise soll ein Student nach Standard 2 in der Lage sein, sich einen „effizienten und effektiven Zugang zu den benötigten Informationen" zu verschaffen, indem er sich unter anderem Kenntnisse über „den Umfang, Inhalt und die Organisation von Retrievalsystemen"39 erarbeitet. Die Standards beabsichtigen laut ACRL nicht, eine Definition für Informationskompetenz festzulegen; sie sollen lediglich die Qualität der Angebote dazu nach „best practice“ gestalten helfen: „The focus is on defining the elements of best practices in information literacy programming " 40 . Zwar liegt noch kein vollständiges Zertifizierungsverfahren vor, doch es ist möglich, einzelne Standards zu prüfen, wenn die Elemente des Schulungsangebots danach ausgerichtet sind. In den curricularen Angeboten zur Informationskompetenz im Bachelor- oder Masterstudium wird im kleineren oder größeren Maße über das Erreichen von Lernzielen gearbeitet. Je nach Studienorganisation ist ein Test obligatorisch, um die erreichbaren Credit Points zu erlangen. Der Bewertungsgehalt der Credit Points ist allerdings mit Vorsicht zu genießen; sie werden über die Hochschullandschaft hinweg unterschiedlich gewichtet vergeben. Dies kann mit oder ohne Benotung sein. Die erreichte Anzahl der Credit Points misst, wie schon ausgeführt, den Workload, ist also eher keine differentielle Bewertung der Leistung oder gar Informationskompetenz.

Lernerfolg bzw. Leistungsergebnisse für Informationskompetenzangebote insgesamt können auch mithilfe von Standards abgesichert werden, die für die Qualifi- kation der Informationsvermittler aufgestellt werden. So hat die ACRL z. B. „Standards for Proficiencies for Instruction Librarians and Coordinators"41 und weitere Vorgaben für Bibliothekare verschiedener Bibliothekstypen erstellt. Maßgeblich von der Deutschen Gesellschaft für Informationswissenschaft und Informationspraxis e.V. (DGI) im Rahmen des Leonardo Da Vinci-Programms der Europäischen Kommission mitgestaltet wurde das „Handbuch der Informationskompetenz" (BID) des European Council of Information ${ }^{42}$, das Standards für Qualifikation und Qualifikationsniveaus von Informationsfachleuten formuliert. Standards in dieser Form sind valide Anhaltspunkte für Qualität und können als Bestandteil eines prozessualen Qualitätsmanagements die Prozess- und Produktqualität der Aufgabe Schulung, Beratung etc. unterstützen.

Nutzung (Effektivität 3) im Sinne der erhöhten Nutzung allgemein sowie vermittelter Informationssysteme oder von Beständen:

- Erhöhung der Anmeldezahlen, Erhöhung der Besucherzahlen, erhöhte Nutzung der Bestände, Ausleihen, erhöhte Anzahl von Datenbankzugriffen, erhöhte Nutzung anderer Informationsmittel.

- Erhöhte Nutzung durch bestimmte Benutzergruppen und aanderer.

Bei dieser Form der Evaluation geht man davon aus, dass Schulungen einen Einfluss auf die Nutzungszahlen haben können. Rein wissenschaftlich gesehen, ist diese Annahme ohne entsprechende Prüfung nicht bewiesen. Praktisch kann man schon mit einigem kritischen Vorbehalt einen solchen Einfluss annehmen. Die Zahlen erfassen indirekt mit dem gleichen kritischen Vorbehalt auch Nutzungskompetenz (Lernerfolg, s. o.).

Nutzerzufriedenheit (Effektivität 4) mit dem Schulungsangebot:

- Zustimmungs- oder Ablehnungsäußerungen während der Veranstaltung, z. B. Aufmerksamkeit, Aktivität oder

${ }^{36}$ American Library Association: Information Literacy Competency Standards for Higher Education. $2000<$ http://www. ala.org/ala/mgrps/divs/acrl/standards/standards.pdf> [Zugriff am 06.11.2008].

37 Vgl. Homann, Benno: Standards der Informationskompetenz: Eine Übersetzung der amerikanischen Standards der ACRL als argumentative Hilfe zur Realisierung der „Teaching Library“. In: Bibliotheksdienst 36 (2002) 5, S. 625-637.

${ }^{38}$ NIK-BW (Anm. 7).

${ }^{39}$ Homann (Anm. 37), hier S. 631.

${ }^{40} \mathrm{Vgl}$. ACRL: Characteristics of Programs of Information Literacy that Illustrate Best Practices: A Guideline. 2003 $<$ http://www.ala.org/ala/mgrps/divs/acrl/standards/characteristics.cfm> [Zugriff am 09.11.2008].

${ }^{41} \mathrm{Vgl}$. ACRL: Standards for Proficiencies for Instruction Librarians and Coordinators. $2007<$ http://www.ala.org/ala/ mgrps/divs/acrl/standards/profstandards.cfm> [Zugriff am 09.11.2008].

$42 \mathrm{Vgl}$. European Council of Information Associations (ECIA): Euroguide: Handbuch für Informationskompetenz (BID). Band 2: Qualifikationsniveaus der europäischen Informationsfachleute. Frankfurt $2004<$ http://www.certidoc.net/de/ euref2-deutsch.pdf> [Zugriff am 09.11.2008]. 
Passivität, Ablenkung, Störverhalten etc. der Teilnehmenden. Hier gilt es, ein Verfahren der Protokollierung zu etablieren (Notizen des Dozenten auf Laufzettel, begleitende Beobachtung des Schulungsverlaufs durch Fachkollegen bzw. Mitglieder der Peer-Group der Teilnehmer).

- Nachfragen der Nutzer nach einer Veranstaltung, erhöhte Nutzung: ein Nutzer kommt mit seiner Spezialfrage wieder, weil er die Bibliothek als „kompetent erlebt hat", Systeme werden häufiger genutzt etc. Natürlich muss hier die Bibliothek für ein geeignetes Erfassungssystem sorgen. Die Frage „woher kennen Sie uns" gehört z. B. in den Ablauf jeder Benutzeranmeldung. Plätze, wo solche Daten aufkommen, müssen entdeckt, die Daten systematisch erfasst werden.

- Beurteilung der Veranstaltungen über einen Evaluationsbogen oder über eine Benutzerumfrage ${ }^{43}$. Dies sind die am häufigsten eingesetzten Methoden.

- Methodische Ansätze der Befragung wie ServQual oder LibQual+T ergründen die Nutzerzufriedenheit in verschiedenen Dimensionen ${ }^{44}$ und liefern zum Teil auch Tools zur Datenanlyse und -darstellung ${ }^{45}$.

Die begleitende kooperative Evaluation durch Beobachtung der Schulung durch einen Fachkollegen oder einen unbeteiligten Dritten (Pseudo-Teilnehmer) erhebt eine Menge unterschiedlicher Daten, nicht nur sichtbare Anzeichen von Lernerfolg, Benutzerzufriedenheit, sondern auch Wahrnehmungen zur Effizienz und Effektivität der Organisation der Schulungen, der Didaktik, der Unterrichtsmaterialien, der Performance des Dozenten etc. Da dieser bei seiner Arbeit von Kollegen oder anderen beobachtet wird und dadurch mittelbar eine Leistungsbewertung im Spiel ist, ist zu gewährleisten, dass über das Verfahren Vertrauen, Sachlichkeit und Fairness sichergestellt sind. Zuständige Gremien sollten beteiligt werden.

In Bezug auf Nutzerzufriedenheit ist zu beachten: „Zufriedenheit und Nutzung“ wie „Zufriedenheit und Lernerfolg" sind grundsätzlich voneinander zu unterscheidende Gegenstände. Die Zufriedenheit hängt genuin mit den Erwartungen des Nutzers im Vergleich mit seiner Wahrnehmung der Leistung zusammen. Als erstes zählt das direkte Interesse des Nutzers „bekomme ich die von mir gewünschte Information“. Außerdem spielen Haltungen und Motive aller Art eine Rolle. Besonders wichtig für die Zufriedenheit ist die Wahrnehmung der „Aufmerksamkeit" und der „Freundlichkeit" des Informationsdienstleisters.

Problematisch sind z. B. unfreiwillige Schulungssituationen, in der das eigentliche Ziel einer Schulung, z. B. kompetent mit einer Recherche umgehen zu lernen, im schlechtesten Fall von den Teilnehmenden gar nicht „eigens" verfolgt wird. Beispielhaft seien hier Veranstaltungen für Schüler zur Vorbereitung der Facharbeit in der 12. Klasse genannt. Hier kann man verschiedene Erfahrungen mit Teilnehmenden sammeln, von aktiv interessiert bis hin zu völlig nörgelig und unzufrieden, da eventuell auch völlig uninteressiert. Dies muss mit dem Angebot oder der Durchführungsqualität bzw. der „Leistung des Dozenten“ nicht unbedingt etwas zu tun haben. Dennoch kann man natürlich Änderungen erwägen (Inhalte, Vermittlung etc.).

Der Unzufriedenheit mit einer Veranstaltung muss nicht notwendig die Nichtnutzung der Einrichtung folgen. Der
Zufriedenheit mit der Veranstaltung kann trotzdem langfristiges Desinteresse folgen, denn letztlich ist immer der tatsächliche, bestimmte Bedarf an Angeboten der Bibliothek entscheidend.

Optimierung des Angebots: Dazu können praktisch alle schon genannten Erhebungen dienen, hier unter dem Aspekt „wie sind die Erfahrungen“ mit dem Angebot, ergänzend:

- dokumentierte Erfahrungssammlungen zur zeitlichen, räumlichen, technischen Organisation, zum Anmeldeverfahren, zur Gruppenzusammensetzung, Gruppengröße, bezüglich Schulungsinhalten (Umfang, Tiefe), Schulungsmaterialien (Kürze, Übersichtlichkeit, Verständlichkeit, Einsetzbarkeit, Variierbarkeit), Erfahrungen des Dozenten (Erfassungsbögen, Laufzettel, die später ausgewertet werden), regelmäßig Prüfung der Bedarfsorientierung.

Hier ist der direkten Frage an die Nutzer/innen, ob Angebote zur Informationskompetenz gewünscht sind, mit Vorsicht zu begegnen. Es gibt kaum Kunden, die diesen Wunsch in dieser allgemeinen Form bejahen, steckt darin doch das Problem, sich etwas eingestehen zu sollen, abgesehen davon, dass nicht jeder Benutzer mit dem Begriff Informationskompetenz etwas anfangen kann. Geeigneter sind Fragen nach konkreten Rechercheproblemen oder -interessen.

\subsection{Prozessorientiertes Qualitätsmanagement}

Die bisher genannten Möglichkeiten der Evaluierung sind eher einzelne, auf ein Element der Leistung oder das Produkt bezogene, punktuelle Fragestellungen. Ganzheitliche Ansätze wie das Indikatorsystem „Balanced Scorecard“ bilden die Bibliothek als Leistungsfeld insgesamt ab. Das von POLL und TE BOEKHORST vorgelegte System zur Leistungsmessung in Bibliotheken bietet als einzelnen Indikator "Attendances at training lessons per Capita"46 (Schulungsbesuche pro Kopf). Letztlich ist jedoch die Gesamtsicht der Interdependenzen verschiedener (auch dort genannter) Indikatoren maßgeblich. Quantitativ ausgerichtete Indikatoren machen mittelbar qualitative Leis-

${ }^{43}$ Allgemeine Hinweise zu Fragebögen etc. bieten z. B. Kirchhoff, Sabine: Der Fragebogen: Datenbasis, Konstruktion und Auswertung. 4. Aufl. Wiesbaden 2008 und Mummendey, Hans-Dieter: Die Fragebogen-Methode. 5. Aufl. Göttingen 2008.

44 Z. B. „affect of service: including empathy of staff, responsiveness, assurance and reliability“, „information control: including the scope of the content made available by the library, convenience, ease of navigation, timeliness, the equipment made available und self-reliance“, vgl. Brophy, Peter: Measuring library performance: principles and techniques. London 2006, S. 45.

${ }^{45} \mathrm{Vgl}$. dazu Association of Research Libraries: Sample Screens from the LibQUAL+ ${ }^{\text {TM }}$ Survey. $2004<$ <ttp://www.libqual.org/ Information/Sample/index.cfm> [Zugriff am 09.11.2008].

${ }^{46}$ Vgl. Poll, Roswitha und Peter te Boekhorst: Measuring Quality: Performance measurement in Libraries. 2. Aufl. München 2007. 
tungen einer Bibliothek, wie sie BROPHY $\mathrm{Y}^{47}$ formuliert hat, messbar und können in der Gesamtsicht das Material für die Gegenstände eines prozessorientierten Evaluierungsansatzes stellen. Standards wie die der ARCL und andere ergänzen dieses Material, indem sie einen Maßstab für die Leistungen liefern. Qualitätssicherung und -prüfung wird so gesehen im Kontext des Gesamtprozesses der Leistungserstellung in Augenschein genommen. Der Gedankengang lautet in etwa: „entspricht jeder Schritt der Leistungserstellung festgelegten Anforderungen bzw. Standards, ist die Qualität insgesamt messbar und optimierbar". Es ergibt sich im besten Fall ein zertifizierbares Gerüst prüfbarer Anforderungen, die im hohen Maße Effizienz und Effektivität der Leistung gewährleisten können.

Prozessorientiertes Qualitätsmanagement kann allerdings nur die Güte der Elemente des Angebots nach best practice und aktuellem Kenntnisstand im Hinblick auf das Ziel Informationskompetenz verankern. Die Prüfung der Wirkung in Bezug auf die individuelle Kompetenz des Nutzers wäre eine Aufgabe der Benutzerforschung, durch deren Ergebnisse die Anforderungen an die Elemente jedoch entsprechend gestaltet werden können. Es zeigt sich insgesamt hier, was das Merkmal von Dienstleistungen im Informationsbereich an sich ist: das Produkt entsteht immer mit dem Nutzer. Informationsangebote und insbesondere "Informationskompetenz" ist kein fertiges Produkt, was gekauft und mitgenommen wird. Der Nutzer ist quasi im Prozess der Leistungserstellung im Moment der Begegnung und im weiteren Umgang damit beteiligt. Auch dies muss sich letztlich in den Qualitätsanforderungen wiederfinden.

Im Bereich von Non-Profit-Dienstleistungen in Kultur und Bildung sind Zertifizierungen nach verschiedenen Qualitätsmanagementsystemen auf dem Vormarsch. Dies ist beispielsweise die Zertifizierung nach ISO $9000 \mathrm{ff}$. oder EFQM. Es gibt auch Verfahren, die sich explizit auf Bereiche des Lernens beziehen, z. B. das DIN-Referenzmodell für Qualitätsmanagement und Qualitätssicherung PAS1031-1 für E-Learning in der Aus- und Weiterbildung ${ }^{48}$ bzw. eine große Zahl von Modellen aus dem Bereich der Evaluation schulischer und beruflicher Bildung.

Eine prozessorientierte Sicht auf Angebote zur Informationskompetenz, insbesondere Schulungen, umfasst verschiedene Leistungsphasen, in denen bestimmte Aspekte betrachtet werden. Die Prüfung erfolgt je nach System protokolliert anhand von Leistungsspezifikationen und -indikatoren, eventuell versehen mit zu erreichenden Bewertungspunkten.

Hier einige Themenkreise, die auch in Auswahl angegangen werden könnten, wenn man das Ziel des prozessualen QM verfolgt:

\section{Qualität bei der Konzeption von Schulungen}

- Sicherung der Kompetenz des Managements: Beschreibung der Anforderungen an die Stelle, Standards für Personal und Personalauswahl.

- Verfahren zur Analyse des Kundenbedarfs, des Marktes, der Kosten, in Hinblick auf Wirtschaftlichkeit, Kundenorientierung, Effizienz und Marktfähigkeit.

- Sicherung fachlicher Qualität: Orientierung an wissenschaftlichen Standards aus der Fachdisziplin und für Informationskompetenz.

- Sicherung didaktischer Qualität: Orientierung an Forschung oder Standards, modernen Lernkonzepten,
Standards zum E-Learning oder Blended Learning, Ausrichtung am Kompetenzlevel der Nutzer bis hin zur Eignungsdiagnostik.

- Sicherung einer adäquaten (marktgerechten) Organisation des Angebotes: Prüfung der Gestaltung des Programms (Module, organisatorische/technische Aspekte) am Kundenbedarf.

- Sicherung der Einhaltung von Sicherheitsvorschriften, bzw. zur Ergonomie und Barrierefreiheit.

- Verfahren der Einführung des Produktes (Implementierung in Echtbetrieb), Weiterentwicklung, (Weiter-) Qualifizierung von Durchführenden.

Qualität bei der Gestaltung von Lernumgebungen und Schulungsmaterialien

- Sicherung der Eignung von Schulungsmaterial: Vorgaben für die Organisation der Materialien, Übersichtlichkeit, Klarheit, Layout, Einsatz.

- Sicherung der Eignung und Qualität von elektronischen Lernplattformen anhand von Standards für E-Learning, Barrierefreiheit, Daten-/Zugriffssicherheit (z. B. DINIZertifizierung eines Servers/Systems).

Qualität bei der Durchführung von Schulungen

- Sicherung der Kompetenz des Personals in Organisation und Lehre (s. o.).

- Sicherung des Verfahrens der Anmeldung, Kundenkontakt, Ansprechbarkeit.

- Sicherung der operativen Planung: Termine, Ort, Technik, Gruppengröße, Barrierefreiheit, Sicherheit.

- Sicherung von Leitung und Betreuung in elektronischen Lernumgebungen usw.

Sicherung der Qualität bei Evalution und Optimierung

- Vorliegen von Systemen zur Evaluation, Leistungsdefinition, Dokumentation.

- Vorliegen von Verfahren zur Optimierung (Diskussion, Information, Planung, Entwicklung).

Sicherung der Qualität von Werbung und Marketing

- Marketing und Öffentlichkeitsarbeit durch Fachleute, Marketingkonzept, zielgerichtete Pressearbeit, klare Organisation der Öffentlichkeitsarbeit im Hause, Einbezug aller Kommunikationssysteme.

${ }^{47}$ Die Merkmale „performance“ gleich „a service meets its most basic purpose“, „reliability“ gleich „consistency of the service's performance in use“, „servicability" gleich „Level of help available to users“, „communication“ gleich „clear explanation of services and options in language free of jargon" etc. sind im wahrsten Sinne des Wortes qualitative und müssen in Bezug auf Schulungen durch genaue Anforderungen konkretisiert werden, denen gegebenenfalls Indikatoren zugeordnet werden. Vgl. Poll, te Boekhorst (Anm. 46) S. 14, zit. n.: Brophy, Peter: The quality of libraries. In: Die effektive Bibliothek: Roswitha Poll zum 65. Geburtstag. München 2004, S. $30 \mathrm{ff}$.

${ }^{48}$ Deutsches Institut für Normung (DIN) (Hrsg.): Qualitätssicherung und Qualitätsmanagement im e-Learning: Beiträge zur Anwendung der PAS 1032-1. Berlin 2006. 


\section{$6 \quad$ Fazit}

Für die Vermittlung von Informationskompetenz durch Hochschulbibliotheken gibt es keinen Königsweg. Zu unterschiedlich sind Infrastruktur und Leitbilder der unterschiedlichen Einrichtungen und ihrer Hochschulen. Aus dem hier vorgestellten Angebot in der Praxis bewährter Modelle sollte es jedoch möglich sein, ausreichend Anregungen und Unterstützung zu beziehen, um dann vor Ort sein individuell auf die eigene Situation und die eigenen Kunden zugeschnittenes Konzept zur erfolgreichen Vermittlung von Informationskompetenz zu generieren.

\section{Anschriften der Autoren:}

Mario Hütte

Fachhochschule Dortmund

University of Applied Sciences and Arts

Hochschulbibliothek

Sonnenstr. 96

D-44139 Dortmund

E-Mail: huette@fhb.fh-dortmund.de
Susanne Kundmüller-Bianchini Hochschul- und Kreisbibliothek Hochschule Bonn-Rhein-Sieg

Grantham-Allee 20

D-53757 Sankt Augustin

E-Mail: susanne.kundmueller@bib.fh-bonn-rhein-sieg.de

Annette Kustos, M. A., M.A.-LIS,

Universitätsbibliothek der FernUniversität in Hagen

Waldweg 2

D-58730 Fröndenberg

E-Mail: annette.kustos@FernUni-Hagen.de

Dr. Annemarie Nilges

Universitäts- und Landesbibliothek Düsseldorf

Universitätsstrasse 1

D-40225 Düsseldorf

E-Mail: Nilges@ub.uni-duesseldorf.de

Dr. Anja Platz-Schliebs

Universitätsbibliothek Wuppertal

Postfach 100127

D-42001 Wuppertal

E-Mail: platz-schliebs@bib.uni-wuppertal.de 